\title{
The Mathai-Quillen Formalism and Topological Field Theory因
}

\author{
Matthias Blau \\ NIKHEF-H \\ P.O. Box 41882, 1009 DB Amsterdam \\ The Netherlands
}

March 9, 1992

\begin{abstract}
These lecture notes give an introductory account of an approach to cohomological field theory due to Atiyah and Jeffrey which is based on the construction of Gaussian shaped Thom forms by Mathai and Quillen. Topics covered are: an explanation of the Mathai-Quillen formalism for finite dimensional vector bundles; the definition of regularized Euler numbers of infinite dimensional vector bundles; interpretation of supersymmetric quantum mechanics as the regularized Euler number of loop space; the Atiyah-Jeffrey interpretation of Donaldson theory; the construction of topological gauge theories from infinite dimensional vector bundles over spaces of connections.
\end{abstract}

NIKHEF-H/92-07

${ }^{*}$ Notes of lectures given at the Karpacz Winter School on 'Infinite Dimensional Geometry in Physics' (17 - 27 February 1992).

$\dagger$ 'e-mail: t75@nikhefh.nikhef.nl, 22747::t75 


\section{Contents}

1 Introduction 2

2 The Mathai-Quillen Formalism 5

2.1 The Euler number of a finite dimensional vector bundle . . . . . . 5

2.2 The Thom class and the Mathai-Quillen form ....... 8

2.3 The Mathai-Quillen formalism for infinite dimensional vector bundles 12

3 The Euler Number of Loop Space and Supersymmetric Quantum Mechanics 15

3.1 Loop space geometry . . . . . . . . . . . . . . . . . 15

3.2 Supersymmetric quantum mechanics . . . . . . . . . . . 16

3.3 The Mathai-Quillen form from supersymmetric quantum mechanics 21

4 The Euler Number of Vector Bundles over $\mathcal{A} / \mathcal{G}$ and Topological Gauge Theory 21

4.1 Geometry of gauge theories . . . . . . . . . . . 22

4.2 The Atiyah-Jeffrey Interpretation of Donaldson theory . . . . . 24

4.3 Flat connections in two and three dimensions . . . . . . 28

References

\section{Introduction}

Topological field theory has been a lively area for research ever since the appearance of the seminal work by Witten [1, 2, 3] a few years ago. Activity in the field increased when the observation was made [4, 5] that topological gravity in two dimensions is closely related to two-dimensional quantum gravity and its description in terms of random matrix models. Several reviews of the subject are now available日.

I will try to complement these existing reviews by focussing on an approach to topological field theory based on the construction by Mathai and Quillen [10] of Gaussian shaped Thom forms for finite dimensional vector bundles. This very elegant approach is due to Atiyah and Jeffrey [11] who realized that topological field theory could be regarded as an infinite dimensional generalization of this construction. There are several advantages of adopting this point of view. First of all, it provides an a priori explanation of the fact that finite dimensional topological invariants can be represented by functional integrals, the hallmark of topological field theory. Moreover, it has the charming property of giving a

\footnotetext{
${ }^{1}$ See [6, 7, 8] for an account of the relation among topological gravity, matrix models, intersection theory on moduli space, and integrable models, and [9] for a general review of topological field theory.
} 
unified description of all kinds of (cohomological) topological field theories and supersymmetric quantum mechanics. This has the added bonus of making this approach quite elementary as it allows one to develop the main ideas in a quantum mechanical setting and to then transfer them almost verbatim to field theory. Lastly, it also provides some insight into the mechanism of the localization of path integrals in supersymmetric quantum mechanics and topological field theory.

To those already familiar with the subject, these lectures will hopefully provide a new and perhaps enlightning perspective on topological field theory. At the same time they should, ideally, constitute an elementary introduction to the subject requiring no prior knowledge of the field and little more than some basic differential geometry and the ability to perform Gaussian integrals.

The recurrent theme in these notes will be the Euler number of a vector bundle. In order to understand the basic idea of the Atiyah-Jeffrey approach, let us therefore recall that classically there exist two quite different prescriptions for calculating the Euler number $\chi(X) \equiv \chi(T X)$ of (the tangent bundle of) a manifold $X$. The first is topological in nature and instructs one to choose a vector field $V$ on $X$ with isolated zeros and to count these zeros with signs (this is the Hopf theorem). The second is differential geometric and represents $\chi(X)$ as the integral over $X$ of a density (top form) $e_{\nabla}$ constructed from the curvature of some connection $\nabla$ on $X$ (the Gauss-Bonnet theorem). Likewise, the Euler number $\chi(E)$ of some other vector bundle $E$ over $X$ can be determined in terms of either a section $s$ of $E$ or a connection $\nabla$ on $E$.

A more general formula, obtained by Mathai and Quillen [10], interpolates between these two classical prescriptions. It relies on the construction of a form $e_{s, \nabla}(E)$ which depends on both a section $s$ and a connection $\nabla$. This form has the property that

$$
\chi(E)=\int_{X} e_{s, \nabla}(E)
$$

for all $s$ and $\nabla$. Moreover, this equation reduces to the Hopf or Gauss-Bonnet theorem for appropriate choice of $s$ (for isolated zeros to the former and to the latter for $s=0)$.

What Atiyah and Jeffrey [11] pointed out was that, although $e_{\nabla}$ and $\int_{X} e_{\nabla}$ do not make sense for infinite dimensional $E$ and $X$, the Mathai-Quillen form $e_{s, \nabla}$ can be used to formally define regularized Euler numbers $\chi_{s}(E)$ of such bundles by

$$
\chi_{s}(E):=\int_{X} e_{s, \nabla}(E)
$$

for certain choices of $s$. Although not independent of $s$, these numbers $\chi_{s}(E)$ are naturally associated with $E$ for natural choices of $s$ and are therefore likely to be of topological interest.

It is precisely such a representation of topological invariants (in a non-technical 
sense2) by functional integrals which is the characteristic property of topological field theories, and which could also be taken as their definition. This suggests, that certain topological field theories can be interpreted or obtained in this way. It will be the main aim of these notes to explain that this is indeed the case for the cohomological theories (i.e. not Chern-Simons theory and its siblings). The models we consider explicitly are, in addition to supersymmetric quantum mechanics, Donaldson theory [1] and various theories of flat connections discussed e.g. in [13, 14, 15] and 116, 17, 18]. This framework is, however, broad enough to include topological sigma models, twisted minimal models, and their coupling to topological gravity as well (see [19, 20]).

The following notes consist of three sections, dealing with the Mathai-Quillen formalism, supersymmetric quantum mechanics, and topological gauge theory respectively. Each section begins with a brief review of the required mathematical background. Thus section 2.1 recalls the classical expressions for the Euler class and Euler number of a finite dimensional vector bundle. For our present purposes the Euler number of a vector bundle is best understood in terms of its Thom class and section 2.2 exlains this concept. It also contains the construction of the Gaussian shaped Thom form of Mathai and Quillen and its descendants $e_{s, \nabla}$. Section 2.3 deals with the application of the Mathai-Quillen formalism to infinite dimensional vector bundles and their regularized Euler number and introduces the examples to be discussed in more detail in the subsequent sections.

Section 3.1 contains the bare essentials of the geometry of the loop space $L M$ of a manifold $M$ necessary to apply the Mathai-Quillen formalism to its tangent bundle. Section 3.2 exlains how supersymmetric quantum mechanics can be interpreted as defining or arising as a path integral representation of the regularized Euler number of $L M$. Some related results like the path integral proofs of the Gauss-Bonnet and Poincaré-Hopf theorems are reviewed in the light of this derivation. In section 3.3 it is shown that the finite dimensional MathaiQuillen form can, in turn, be derived from supersymmetric quantum mechanics.

Section 4.1 deals with the geometry of gauge theories. We derive an expression for the curvature form of the principal fibration $\mathcal{A} \rightarrow \mathcal{A} / \mathcal{G}$ and give a formula for the Riemann curvature tensor of moduli subspaces $\mathcal{M} \subset \mathcal{A} / \mathcal{G}$. We also introduce those infinite dimensional bundles which will enter into the subsequent discussion of topological gauge theory. In section 4.2 it is shown that the partition function of Donaldson theory can be interpreted as the regularized Euler number of a bundle of self-dual two-forms over $\mathcal{A} / \mathcal{G}$. It also contains a brief discussion of

\footnotetext{
${ }^{2}$ What is meant by 'topological' in this context is the invariance of numbers like $\chi_{s}(E)$ under deformations of certain of the data entering into its calculation. It is in this sense that the Donaldson invariants of four-manifolds [12], which arise as correlation functions of the field theory considered in [1], are topological as they are independent of the metric which enters into the definition of the instanton moduli space. They are, however, not topological invariants in the mathematical sense as they have the remarkable property of depending on the differentiable structure of the four-manifold.
} 
some properties of topological field theories in general, as well as some remarks on the interpretation of observables in the present setting. Topological gauge theories of flat connections in two and three dimensions are the subject of section 4.3. In particular, in $3 d$ we sketch the construction of a topological gauge theory representing the Euler characteristic of the moduli space of flat connections; once directly from the tangent bundle of $\mathcal{A} / \mathcal{G}$ and once from supersymmetric quantum mechanics on $\mathcal{A} / \mathcal{G}$. We also construct a two-dimensional analogue of Donaldson theory representing intersection theory on moduli spaces of flat connections.

The basic references for section 2.1 and 2.2 are Bott and Tu [21] and Mathai and Quillen [10]. For section 2.3 see [11] and [17]. The main result of section 4.2 is due to Atiyah and Jeffrey [11], and a detailed discussion of Donaldson theory [1, 12] can be found in [9, pp. 198-247]. Sections 3.2, 3.3 and 4.3 are based on joint work with George Thompson [16, 17, 18]. Further references can be found in the text and further information on topological field theory in the cited reviews and the lectures of Danny Birmingham [22] at this School.

\section{The Mathai-Quillen Formalism}

In section 2.1 we wil recall some well known facts and theorems concerning the Euler class and the Euler number of a finite dimensional vector bundle $E$. For our present purposes the Euler class is most profitably understood in terms of the Thom class of $E$ and we will adopt this point of view in section 2.2. There we also introduce and discuss at some length the Mathai-Quillen formalism which provides, among other things, a concrete differential form realization of the Thom class. In section 2.3 we explain how the Mathai-Quillen formalism can be used to define certain regularized Euler numbers of $E$ when $E$ is infinite dimensional. We will also introduce the examples (supersymmetric quantum mechanics, topological gauge theory) which will then occupy us in the remainder of these notes.

\subsection{The Euler number of a finite dimensional vector bun- dle}

Consider a real vector bundle $\pi: E \rightarrow X$ over a manifold $X$. We will assume that $E$ and $X$ are orientable, $X$ is compact without boundary, and that the rank (fibre dimension) of $E$ is even and satisfies $r k(E)=2 m \leq \operatorname{dim}(X)=n$.

The Euler class of $E$ is an integral cohomology class $e(E) \in H^{2 m}(X, \mathbf{R}) \equiv$ $H^{2 m}(X)$. For $m=1$ (a two-plane bundle) $e(E)$ can e.g. be defined in a rather pedestrian manner (cf. 21] for the material covered in this and the first part of the following section). We choose a cover of $X$ by open sets $U_{\alpha}$ and denote by $g_{\alpha \beta}: U_{\alpha} \cap U_{\beta} \rightarrow S O(2)$ the transition functions of $E$ satisfying the cocycle condition

$$
g_{\alpha \beta}=g_{\beta \alpha}^{-1}, \quad g_{\alpha \beta} g_{\beta \gamma}=g_{\alpha \gamma} .
$$


Identifying $S O(2) \sim U(1)$, we set $\varphi_{\alpha \beta}=i \log g_{\alpha \beta}$ with

$$
\varphi_{\alpha \beta}+\varphi_{\beta \gamma}-\varphi_{\alpha \gamma} \in 2 \pi \mathbf{Z},
$$

so that $d \varphi$ is an additive cocycle,

$$
d \varphi_{\alpha \beta}+d \varphi_{\beta \gamma}=d \varphi_{\alpha \gamma} .
$$

In fact, more than that is true. By introducing a partition of unity subordinate to $\left\{U_{\alpha}\right\}$, i.e. a set of functions $\rho_{\alpha}$ satisfying

$$
\sum_{\alpha} \rho_{\alpha}=1, \quad \operatorname{supp}\left(\rho_{\alpha}\right) \subset U_{\alpha},
$$

and defining one-forms $\xi_{\alpha}$ on $U_{\alpha}$ by $\xi_{\alpha}=(2 \pi)^{-1} \sum_{\gamma} \rho_{\gamma} d \varphi_{\gamma \alpha}$ one finds that

$$
\frac{1}{2 \pi} d \varphi_{\alpha \beta}=\xi_{\beta}-\xi_{\alpha}
$$

which obviously implies (2.3). Thus $d \xi_{\alpha}=d \xi_{\beta}$ on the overlaps $U_{\alpha} \cap U_{\beta}$ and therefore the $d \xi$ 's piece together to give a global two-form on $X$ which is closed but not necessarily exact. The cohomology class of this form is independent of the choice of $\xi$ 's satisfying (2.5) and is the Euler class $e(E) \in H^{2}(X)$ of $E$.

For higher rank bundles a similar construction is possible in principle but becomes rather unwieldy. Fortunately there are other, more transparent, ways of thinking about $e(E)$.

The first of these is in terms of sections of $E$. In general, a twisted bundle will have no nowhere-vanishing non-singular sections and one defines the Euler class to be the homology class of the zero locus of a generic section of $E$. Its Poincaré dual is then a cohomology class in $H^{2 m}(X)$.

The second makes use of the Chern-Weil theory of curvatures and characteristic classes and produces an explicit representative $e_{\nabla}(E)$ of $e(E)$ in terms of the curvature $\Omega_{\nabla}$ of a connection $\nabla$ on $E$. Thinking of $\Omega_{\nabla}$ as a matrix of two-forms one has

$$
e_{\nabla}(E)=\frac{1}{(2 \pi)^{m}} \operatorname{Pf}\left(\Omega_{\nabla}\right)
$$

where $\operatorname{Pf}(A)$ denotes the Pfaffian of the real antisymmetric matrix $A$,

$$
\operatorname{Pf}(A)=\frac{(-1)^{m}}{2^{m} m !} \sum \epsilon_{a_{1} \cdots a_{2 m}} A_{a_{1} a_{2}} \ldots A_{a_{2 m-1} a_{2 m}},
$$

satisfying $\operatorname{Pf}(A)^{2}=\operatorname{det}(A)$. Standard arguments show that the cohomology class of $e_{\nabla}$ is independent of the choice of $\nabla$.

Finally, the third is in terms of the Thom class of $E$ and we will describe this in section 2.2 . 
If the rank of $E$ is equal to the dimension of $X$ (e.g. if $E=T X$, the tangent bundle of $X$ ) then $H^{2 m}(X)=H^{n}(X)=\mathbf{R}$ and nothing is lost by considering, instead of $e(E)$, its evaluation on (the fundamental class $[X]$ of) $X$, the Euler number

$$
\chi(E)=e(E)[X] .
$$

In terms of the two descriptions of $e(E)$ given above, this number can be obtained either as the number of zeros of a generic section $s$ of $E$ (which are now isolated) counted with multiplicity,

$$
\chi(E)=\sum_{x_{k}: s\left(x_{k}\right)=0} \nu_{s}\left(x_{k}\right)
$$

(here $\nu_{s}\left(x_{k}\right)$ is the degree or index of $s$ at $x_{k}$ ), or as the integral

$$
\chi(E)=\int_{X} e_{\nabla}(E) .
$$

Of particular interest to us is the case where $E=T X$. The Euler number $\chi(T X)$ is then equal to the Euler characteristic $\chi(X)$ of $X$,

$$
\chi(T X)=\chi(X) \equiv \sum_{k}(-1)^{k} b_{k}(X)
$$

where $b_{k}(X)=\operatorname{dim}\left(H^{k}(X)\right)$ is the $k^{\prime}$ th Betti number of $X$. In this context, equations (2.9) and (2.10), expressing $\chi(X)$ as the number of zeros of a vector field and the integral of a density constructed from the Riemannian curvature tensor $\mathcal{R}_{X}$ of $X$, are known as the Poincaré-Hopf theorem and the Gauss-Bonnet theorem respectively. For example, in two dimensions $(n=2)$, (2.10) reduces to the well known formula

$$
\chi(X)=\frac{1}{4 \pi} \int_{X} \sqrt{g} d^{2} x R
$$

where $R$ is the scalar curvature of $X$.

For $E=T X$ there is also an interesting generalization of (2.9) involving a vector field $V$ with a zero locus $X_{V}$ which is not necessarily zero-dimensional. Denoting the connected components of $X_{V}$ by $X_{V}^{(k)}$, this generalization reads

$$
\chi(X)=\sum_{k} \chi\left(X_{V}^{(k)}\right) .
$$

This reduces to (2.9) when the $X_{V}^{(k)}$ are isolated points and is an identity when $V$ is the zero vector field.

One of the beauties of the Mathai-Quillen formalism, to be discussed next, is that it provides a corresponding generalization of (2.10), i.e. an explicit differential form representative $e_{s, \nabla}$ of $e(E)$ depending on both a section $s$ of $E$ and a connection $\nabla$ on $E$ such that

$$
\chi(E)=\int_{X} e_{s, \nabla}(E)
$$


and such that (2.13) reduces to any of the above equations for the appropriate choice of $E$ and $s$ (i.e. to (2.10) if $s$ is the zero section, to (2.9) when the zeros of $s$ are isolated, and to (2.12) for a general vectorfield on $T X)$.

If $n>2 m$, then we cannot evaluate $e(E)$ on $[X]$ as in (2.8). We can, however, evaluate it on homology $2 m$-cycles or (equivalently) take the product of $e(E)$ with elements of $H^{n-2 m}(X)$ and evaluate this on $[X]$. In this way one obtains intersection numbers of $X$ associated with the vector bundle $E$. A corresponding interpretation of the Donaldson polynomials [12] as observables in the topological gauge theory of [1] has been given by Atiyah and Jeffrey [11] (cf. section 4.2).

\subsection{The Thom class and the Mathai-Quillen form}

The Euler class $e(E)$ has the property that it is the pullback of a cohomology class on $E$, called the Thom class $\Phi(E)$ of $E$, via the zero section $i: X \rightarrow E$,

$$
e(E)=i^{*} \Phi(E) \text {. }
$$

We will show this explicitly below (cf. equations (2.33,2.34)). To understand the origin and significance of $\Phi(E)$, recall that there are two natural notions of cohomology for differential forms on a vector bundle $E$ over a compact manifold $X$ : ordinary de Rham cohomology $H^{*}(E)$ and compact vertical cohomology $H_{c v}^{*}(E)$. The latter deals with forms whose restriction to any fibre has compact support. As $E$ is contractible to $X$ one has

$$
H^{*}(E) \simeq H^{*}(X)
$$

On the other hand, as the compact cohomology of a vector space only has a generator in the top dimensions (a 'bump' volume form with unit volume), one has

$$
H_{c v}^{*}(E) \simeq H_{c v}^{*-2 m}(X) .
$$

More technically, for forms of compact vertical support one has the notion of 'push-down' or 'integration along the fibres', denoted by $\pi_{*}$. In local coordinates, and for trivial bundles, this is the obvious operation of integrating over the fibres the part of $\omega \in \Omega_{c v}^{*}(E)$ (the space of forms with compact vertical support) which contains a vertical $2 m$-form and interpreting the result as a form on $X$. This prescription gives a globally well defined operation

$$
\pi_{*}: \Omega_{c v}^{*}(E) \rightarrow \Omega^{*-2 m}(X) .
$$

In particular, for any $\omega \in \Omega_{c v}^{*}(E)$ and $\alpha \in \Omega^{*}(X)$ one has

$$
\pi_{*}\left(\left(\pi^{*} \alpha\right) \omega\right)=\alpha \pi_{*} \omega
$$

$\pi_{*}$ commutes with the exterior derivatives on $E$ and $X$ (it is sufficient to check this in local coordinates),

$$
\pi_{*} d_{E}=d_{X} \pi_{*}
$$


and induces the so called Thom isomorphism $\mathcal{T}_{E}: H^{*}(X) \rightarrow H_{c v}^{*+2 m}(E)$ 2.16). Under this isomorphism, the generator $1 \in H^{0}(X)$ corresponds to a $2 m$-dimensional cohomology class on $E$, the Thom class $\Phi(E)$,

$$
\Phi(E)=\mathcal{T}_{E}(1) \in H_{c v}^{2 m}(E)
$$

By definition, $\Phi(E)$ satisfies $\pi_{*} \Phi(E)=1$, so that by (2.18) the Thom isomorphism is explicitly given by

$$
\mathcal{T}_{E}(\alpha)=\left(\pi^{*} \alpha\right) \Phi(E)
$$

After this small digression let us now return to the Euler class $e(E)$ and equation (2.14). As any two sections of $E$ are homotopic as maps from $X$ to $E$, and as homotopic maps induce the same pullback map in cohomology, we can use any section $s$ of $E$ instead of the zero section to pull back $\Phi(E)$ to $X$ and still find

$$
s^{*} \Phi(E)=e(E) \text {. }
$$

The advantage of this way of looking at the Euler class $e(E)$ should now be evident: provided that we can find an explicit differential form representative $\Phi_{\nabla}(E)$ of $\Phi(E)$, depending on a connection $\nabla$ on $E$, we can pull it back to $X$ via a section $s$ to obtain a $2 m$-form

$$
e_{s, \nabla}(E)=s^{*} \Phi_{\nabla}(E)
$$

representing the Euler class $e(E)$ and (if $n=2 m$ ) satisfying (2.13). It should be borne in mind, however, that by (2.22) all these forms are cohomologous so that this construction, as nice as it is, is not very interesting from the cohomological point of view. To get something really new one should therefore consider situations where the forms (2.23) are not necessarily cohomologous to $e_{\nabla}$. As pointed out by Atiyah and Jeffrey [11], such a situation occurs when one considers infinite dimensional vector bundles where $e_{\nabla}$ (an 'infinite-form') is not defined at all. In that case the added flexibility in the choice of $s$ becomes crucial and opens up the pssibility of obtaining well-defined, but $s$-dependent, 'Euler classes' of $E$. We will explain this in section 2.3.

To proceed with the construction of $\Phi_{\nabla}$, let us make two preliminary remarks. The first is that for explicit formulae it is convenient to switch from working with forms with compact support along the fibres to working with 'Gaussian shaped' form rapidly decreasing along the fibres (in a suitable technical sense). Everything we have said so far goes through in that setting [10] and we will henceforth replace $\Omega_{c v}^{*}(E)$ by $\Omega_{r d}^{*}(E)$ etc.

The second is that Pfaffians (2.7) arise as fermionic (Berezin) integrals (this may sound like a rather mysterious remark to make at this point, but is of course one of the reasons why what we are going through here has anything to do with supersymmetry and topological field theory). More precisely, if we have a real 
antisymmetric matrix $\left(A_{a b}\right)$ and introduce real Grassmann odd variables $\chi^{a}$, then

$$
\operatorname{Pf}(A)=\int d \chi e^{\chi^{a} A_{a b} \chi^{b} / 2} .
$$

In particular, we can therefore write the form $e_{\nabla}(2.6)$ as

$$
e_{\nabla}(E)=(2 \pi)^{-m} \int d \chi e^{\chi_{a} \Omega_{\nabla}^{a b} \chi_{b} / 2} .
$$

The idea is now to extend the right hand side of (2.25) to a form $\Phi_{\nabla}(E)$ on $E$ having Gaussian decay along the fibres and satisfying $\pi_{*} \Phi_{\nabla}(E)=1$.

Regarding $E$ as a vector bundle associated to a principal $G$ bundle $P$ with standard fibre $F, E=P \times_{G} F$, we can represent forms on $E$ by basic, i.e. horizontal and $G$-invariant, forms on $P \times F$,

$$
\Omega^{*}(E)=\Omega_{\text {bas }}^{*}(P \times F)
$$

and sections of $E$ by $G$-equivariant maps from $P$ to $F$. Moreover, via the projection $\pi: P \rightarrow X, E$ pulls back to the canonically trivial vector bundle $\pi^{*} E=P \times F$ over $P$ whose induced connection and curvature we also denote by $\nabla$ and $\Omega_{\nabla}$. With this identification understood, the Thom form $\Phi_{\nabla}(E)$ of Mathai and Quillen is given by

$$
\Phi_{\nabla}(E)=(2 \pi)^{-m} e^{-\xi^{2} / 2} \int d \chi e^{\chi_{a} \Omega_{\nabla}^{a b} \chi_{b} / 2+i \nabla \xi^{a} \chi_{a}}
$$

where we have chosen a fixed fibre metric on $F, \xi^{a}$ are coordinates on $F$ and $\nabla \xi^{a}$ is the exterior covariant derivative of $\xi^{a}$, a one-form on $P \times F$. We now check that $\Phi_{\nabla}(E)$ really represents the Thom class of $E$.

First of all, integrating out $\chi$ one sees that (2.27) defines a $2 m$-form on $P \times F$. This form is indeed basic and represents a closed $2 m$-form on $E$. $G$-invariance and horizontality are almost obvious from (2.27) as $\Omega_{\nabla}$ and $\nabla \xi$ are horizontal (by the definition of the covariant exterior derivative). Less evident is the fact that $\Phi_{\nabla}(E)$ is closed. This is best understood in terms of the equivariant cohomology $H_{G}^{*}(F)$ of $F$ (cf. sections 5 and 6 of [10]) and is related to the fact that the exponent in (2.27),

$$
-\xi^{2} / 2+\chi_{a} \Omega_{\nabla}^{a b} \chi_{b} / 2+i \nabla \xi^{a} \chi_{a}
$$

is invariant under the graded (i.e. super-) symmetry

$$
\begin{aligned}
\delta \chi_{a} & =i \xi_{a} \\
\delta \xi^{a} & =\nabla \xi^{a}
\end{aligned}
$$

mapping the Grassmann odd $\chi$ to the even $\xi$ and $\xi$ to the Grassmann odd oneform $\nabla \xi$. 'On shell', i.e. using the $\chi$ equation of motion $i \nabla \xi^{a}=\Omega_{\nabla}^{a b} \chi_{b}$, this supersymmetry squares to rotations by the curvature matrix $\Omega_{\nabla}$,

$$
\begin{aligned}
\delta^{2} \chi^{a} & =\Omega_{\nabla}^{a b} \chi_{b} \\
\delta^{2} \xi^{a} & =\Omega_{\nabla}^{a b} \xi_{b}
\end{aligned}
$$


which is the hallmark of equivariant cohomology. For a more thorough discussion of the relation between the classical (Cartan-, Weil-) models of equivariant cohomology and the BRST model, as well as of the Mathai-Quillen formalism in that context, see [23].

By introducing a Grassmann even scalar field $B_{a}$ with $\delta \chi_{a}=B_{a}$ and $\delta B^{a}=$ $\Omega_{\nabla}^{a b} \chi_{b}$ the 'action' (2.28) becomes $\delta$-exact off-shell,

$$
(2.28) \approx \delta\left(\chi_{a}\left(i \xi_{a}-B^{a} / 2\right)\right.
$$

It is of course no coincidence that the structure we have uncovered here is reminiscent of topological field theory, see e.g. (3.11,4.20) below.

Because of the factor $e^{-\xi^{2} / 2}$, (2.27) is certainly rapidly decreasing along the fibre directions. What remains to be checked to be able to assert that $\Phi_{\nabla}(E)$ represents the Thom class $\Phi(E)$ is that $\pi_{*} \Phi_{\nabla}(E)=1$ or, under the isomorphism (2.26), that $\int_{F} \Phi_{\nabla}(E)=1$. Extracting from the $2 m$-form $\Phi_{\nabla}(E)$ the part which is a $2 m$-form on $F$ we find that indeed

$$
\begin{aligned}
\int_{F} \Phi_{\nabla}(E) & =(2 \pi)^{-m} \int_{F} e^{-\xi^{2} / 2} \int d \chi \frac{\left(i d \xi^{a} \chi_{a}\right)^{2 m}}{2 m !} \\
& =(2 \pi)^{-m} \int_{F} e^{-\xi^{2} / 2} d \xi^{1} \ldots d \xi^{2 m}=1 .
\end{aligned}
$$

This proves that

$$
\left[\Phi_{\nabla}(E)\right]=\Phi(E) \in H_{r d}^{2 m}(E) .
$$

We now take a closer look at the forms $s^{*} \Phi_{\nabla}(E)=e_{s, \nabla}(E)(2.23)$ for various choices of $s$. In our notation $e_{s, \nabla}(E)$ is obtained from (2.27) by replacing the fibre coordinate $\xi$ by $s(x)$. The first thing to note is that for the zero section $i$, (2.27) reduces to (2.25) and therefore

$$
e_{\nabla}(E)=i^{*} \Phi_{\nabla}(E) .
$$

This is a refinement of (2.14) to an equality between differential forms and therefore, in particular, finally proves (2.14) itself.

If $n=2 m$ and $s$ is a generic section of $E$ transversal to the zero section, then we can calculate $\int_{X} e_{s, \nabla}(E)$ by replacing $s$ by $\gamma s$ for $\gamma \in \mathbf{R}$ and evaluating the integral in the limit $\gamma \rightarrow \infty$. In that limit the curvature term in (2.27) will not contribute and one can use the stationary phase approximation to reduce the integral to a sum of contributions from the zeros of $s$, reproducing equation (2.9). The calculation is entirely analogous to similar calculations in supersymmetric quantum mechanics (see e.g. [9]) and I will not repeat it here. In fact, as we will later derive the Mathai-Quillen formula (2.27) from supersymmetric quantum mechanics (section 3.3), this shows that the required manipulations are not only entirely analogous to but identical with those in supersymmetric quantum mechanics. As we could equally well have put $\gamma=0$ in the above, this also establishes directly the equality of (2.9) and (2.10). 
Finally, if $E=T X$ and $V$ is a non-generic section of $X$ with zero locus $X_{V}$, the situation is a little bit more complicated. It turns out that in this case $\int_{X} e_{V, \nabla}$ can be expressed in terms of the Riemann curvature tensor $\mathcal{R}_{X_{V}}$ of $X_{V}$. Here $\mathcal{R}_{X_{V}}$ arises from the data $\mathcal{R}_{X}$ and $V$ entering $e_{V, \nabla}$ via the Gauss-Codazzi equations. Quite generally, these express the curvature $\mathcal{R}_{Y}$ of a submanifold $Y \subset X$ in terms of $\mathcal{R}_{X}$ and the extrinsic curvature of $Y$ in $X$ (we will recall these equations in section 4.1). Then equation (2.12) is reproduced in the present setting in the form (we assume that $X_{V}$ is connected - this is for notational simplicity only)

$$
\chi(X)=\int_{X} e_{V, \nabla}=(2 \pi)^{-\operatorname{dim}\left(X_{V}\right) / 2} \int_{X_{V}} \operatorname{Pf}\left(\mathcal{R}_{X_{V}}\right) .
$$

Again the manipulations required to arrive at (2.35) are exactly as in supersymmetric quantum mechanics [17, 18] and we will perform such a calculation in the context of topological gauge theory in section 4.3 (see the calculations leading to (4.31)).

\subsection{The Mathai-Quillen formalism for infinite dimen- sional vector bundles}

Let us recapitulate briefly what we have achieved so far. Using the MathaiQuillen form $\Phi_{\nabla}(E)$ (2.27), we have constructed a family of differential forms $e_{s, \nabla}(E)$ parametrized by a section $s$ and a connection $\nabla$ and all representing the Euler class $e(E) \in H^{2 m}(X)$. In particular, for $E=T X$, the equation $\chi(X)=$ $\int_{X} e_{V, \nabla}(X)$ interpolates between the classical Poincaré-Hopf and Gauss-Bonnet theorems.

To be in a situation where the forms $e_{s, \nabla}$ are not necessarily all cohomologous to $e_{\nabla}$, and where the Mathai-Quillen formalism thus 'comes into its own' [11], we now consider infinite dimensional vector bundles. To motivate the concept of regularized Euler number of such a bundle, to be introduced below, recall equation (2.12) for the Euler number $\chi(X)$ of a manifold $X$ which we repeat here for convenience in the form

$$
\chi(X)=\chi\left(X_{V}\right) .
$$

When $X$ is finite dimensional this is an identity, while its left hand side is not defined when $X$ is infinite dimensional. Assume, however, that we can find a vector field $V$ on $X$ whose zero locus is a finite dimensional submanifold of $X$. Then the right hand side of (2.36) is well defined and we can use it to tentatively define a regularized Euler number $\chi_{V}(X)$ as

$$
\chi_{V}(X):=\chi\left(X_{V}\right) \text {. }
$$

By (2.13) and the standard localization arguments, as reflected e.g. in (2.35), we expect this number to be given by the (functional) integral

$$
\chi_{V}(X)=\int_{X} e_{V, \nabla}(X) .
$$


This equation can (formally) be confirmed by explicit calculation. The idea is again to replace $V$ by $\gamma V$, so that (2.38) localizes to the zeros of $V$ as $\gamma \rightarrow \infty$, and to show that in this limit the surviving terms in (2.28) give rise to the Riemann curvature tensor of $X_{V}$, expressed in terms of $\mathcal{R}_{X}$ and $V$ via the GaussCodazzi equations. A rigorous proof can probably be obtained in some cases by probabilistic methods as used e.g. by Bismut [24, 25] in related contexts. We will, however, content ourselves with verifying (2.38) in some examples below.

More generally, we are now led to define the regularized Euler number $\chi_{s}(E)$ of an infinite dimensional vector bundle $E$ as

$$
\chi_{s}(E):=\int_{X} e_{s, \nabla}(E) .
$$

Again, this expression turns out to make sense when the zero locus of $s$ is a finite dimensional manifold $X_{s}$, in which case $\chi_{s}(E)$ is the Euler number of some finite dimensional vector bundle over $X_{s}$ (a quotient bundle of the restriction $\left.E\right|_{X_{s}}$, cf. [19, 20]).

Of course, there is no reason to expect $\chi_{s}(E)$ to be independent of $s$, even if one restricts one's attention to those sections $s$ for which the integral (2.39) exists. However, if $s$ is a section of $E$ naturally associated with $E$ (we will see examples of this below), then $\chi_{s}(E)$ is also naturally associated with $E$ and can be expected to carry interesting topological information. This is indeed the case.

It is precisely such a representation of finite dimensional topological invariants by infinite dimensional integrals which is the characteristic property of topological field theories. It is then perhaps not too surprising anymore at this point, that topological field theory actions can be constructed from (2.28) for suitable choices of $X, E$, and $s$.

Here is a survey of the examples we will discuss in a little more detail in the following sections ( $L M$ denotes the loop space of a manifold $M$ and $\mathcal{A}^{k} / \mathcal{G}^{k}$ a space of gauge orbits in $k$ dimensions).

Example $1 X=L M, E=T X, V=\dot{x}$ (section 3.2)

(2.28) becomes the standard action $S_{M}$ of de Rham supersymmetric quantum mechanics and

$$
\int_{L M} e_{V, \nabla}(L M)=Z\left(S_{M}\right)
$$

is the partition function of $S_{M}$. The zero locus $(L M)_{V}$ of $V$ is the space of constant loops, i.e. $(L M)_{V} \simeq M$. We therefore expect (2.40) to calculate

$$
\chi_{V}(L M)=\chi(M) .
$$

As this indeed agrees with the well known explicit evaluation of $Z\left(S_{M}\right)$ in the form

$$
Z\left(S_{M}\right)=(2 \pi)^{-\operatorname{dim}(M) / 2} \int_{M} \operatorname{Pf}\left(\mathcal{R}_{M}\right),
$$


this is our first confirmation of (2.39). Conversely the Mathai-Quillen formalism now provides an understanding and explanation of the mechanism by which the (path) integral (2.40) over $L M$ localizes to the integral (2.42) over $M$.

Instead of the vector field $\dot{x}$ one can also use $\dot{x}+W^{\prime}$, where $W^{\prime}$ denotes the gradient vectorfield of some function $W$ on $M$. By an argument to be introduced in section 3 (the 'squaring argument') the zero locus of this vector field is the zero locus of $W^{\prime}$ on $M$ (i.e. $\dot{x}=W^{\prime}=0$ ) whose Euler number is the same as that of $M$ by (2.36),

$$
\chi_{V}(L M)=\chi\left(M_{W^{\prime}}\right)=\chi(M) .
$$

Again this agrees with the explicit evaluation of the path integral of the corresponding supersymmetric quantum mechanics action.

Example $2 X=\mathcal{A}^{4} / \mathcal{G}^{4}, E=\mathcal{E}_{+}, s=\left(F_{A}\right)_{+}($section 4.2$)$

$\left(\mathcal{E}_{+}\right.$is a certain bundle of self-dual two-forms over $\mathcal{A}^{4} / \mathcal{G}^{4}$ and $\left(F_{A}\right)_{+}$is the selfdual part of the curvature $F_{A}$ of $A$ ). The zero locus $X_{s}$ is the moduli space $\mathcal{M}_{I}$ of instantons, and not unexpectedly the corresponding action is that of Donaldson theory [12, 1]. The partition function $\chi_{s}\left(\mathcal{E}_{+}\right)$is what is known as the first Donaldson invariant and is only non-zero when $d(\mathcal{M}) \equiv \operatorname{dim}\left(\mathcal{M}_{I}\right)=0$. If $d(\mathcal{M}) \neq 0$ then one has to insert elements of $H^{d(\mathcal{M})}\left(\mathcal{A}^{4} / \mathcal{G}^{4}\right)$ into the path integral in the manner explained at the end of section 2.1 to obtain non-vanishing results (the Donaldson polynomials). This interpretation of Donaldson theory is due to Atiyah and Jeffrey [11].

Example $3 X=\mathcal{A}^{3} / \mathcal{G}^{3}, E=T X, V=* F_{A}$ (section 4.3) ( $*$ is the Hodge operator, and the one-form $* F_{A}$ defines a vector field on $\mathcal{A}^{3} / \mathcal{G}^{3}$, the gradient vector field of the Chern-Simons functional). The zero locus of $V$ is the moduli space $\mathcal{M}^{3}$ of flat connections and the action coincides with that constructed in 113, 14, 17]. Again one finds full agreement of

$$
\chi_{V}\left(\mathcal{A}^{3} / \mathcal{G}^{3}\right)=\chi\left(\mathcal{M}^{3}\right)
$$

with the partition function of the action which gives $\chi\left(\mathcal{M}^{3}\right)$ in the form (2.35), i.e. via the Gauss-Codazzi equations for the embedding $\mathcal{M}^{3} \subset \mathcal{A}^{3} / \mathcal{G}^{3}$. In 11 this partition function was first identified with a regularized Euler number of $\mathcal{A}^{3} / \mathcal{G}^{3}$. We have now identified it more specifically with the Euler number of $\mathcal{M}^{3}$. In [26] it was shown that for certain three-manifolds (homology spheres) $\chi_{V}\left(\mathcal{A}^{3} / \mathcal{G}^{3}\right)$ is the Casson invariant. Hence our considerations suggest that the Casson invariant can be defined as $\chi\left(\mathcal{M}^{3}\right)$ for more general three-manifolds [17].

Example $4 X=L\left(\mathcal{A}^{3} / \mathcal{G}^{3}\right), E=T X, V=\dot{A}+* F_{A}$ (section 4.3)

This is supersymmetric quantum mechanics on $\mathcal{A}^{3} / \mathcal{G}^{3}$ and in a sense a combination of all the three above examples. The resulting (non-covariant) gauge theory action in $3+1$ dimensions is that of Donaldson theory (example 2). After partial localization from $L\left(\mathcal{A}^{3} / \mathcal{G}^{3}\right)$ to $\mathcal{A}^{3} / \mathcal{G}^{3}$ it is seen to be equivalent to the action of example 3. Further reduction to the zeros of the gradient vector field $* F_{A}$ (example 1) reduces the partition function to an integral over $\mathcal{M}^{3}$ and calculates 
$\chi\left(\mathcal{M}^{3}\right)$. This again confirms the equality of the left and right hand sides of (2.38). The reason why Donaldson theory is related to instanton moduli spaces in example 2, but to moduli spaces of flat connections in this example is explained in [17].

\section{The Euler Number of Loop Space and Su- persymmetric Quantum Mechanics}

In this section we will work out some of the details of example 1. We begin with a (very) brief survey of the geometry of loop space (section 3.1). We then apply the Mathai-Quillen formalism to the tangent bundle of loop space, derive supersymmetric quantum mechanics from that, and review some of the most important features of supersymmetric quantum mechanics in the light of this derivation (section 3.2). Finally, to complete the picture, we explain how the finite-dimensional Mathai-Quillen form (2.27) can be derived from supersymmetric quantum mechanics (section 3.3).

\subsection{Loop space geometry}

We denote by $M$ a smooth orientable Riemannian manifold with metric $g$ and by $L M$ the loop space of $M$, i.e. the space of smooth maps from the circle $S^{1}$ to $M$,

$$
L M:=C^{\infty}\left(S^{1}, M\right)
$$

(consistent with the sloppyness to be encountered throughout these notes we will not worry about the technicalities of infinite dimensional manifolds). Elements of $L M$ are denoted by $x(t)$ or $x^{\mu}(t)$, where $t \in[0,1], x^{\mu}$ are (local) coordinates on $M$ and $x^{\mu}(0)=x^{\mu}(1)$. In supersymmetric quantum mechanics it is convenient to scale $t$ such that $t \in[0, \beta]$ and $x^{\mu}(0)=x^{\mu}(\beta)$ for some $\beta \in \mathbf{R}$, and to regard $\beta$ as an additional parameter (the inverse temperature) of the theory.

A tangent vector to a loop $x(t)$ can be regarded as an infinitesimal variation of the loop. As such it can be thought of as a vector field on the image $x\left(S^{1}\right) \subset M$ (tangent to $M$ but not necessarily to the loop $x\left(S^{1}\right)$ ). In other words, the tangent space $T_{x}(L M)$ to $L M$ at the loop $x(t)$ is the space of smooth sections of the tangent bundle $T M$ restricted to the loop $x(t)$,

$$
T_{x}(L M) \simeq \Gamma^{\infty}\left(x^{*}(T M)\right)
$$

There is a canonical vector field on $L M$ which generates rigid rotations $x(t) \rightarrow$ $x(t+\epsilon)$ of the loop around itself. It is given by $V(x)(t)=\dot{x}(t)$ (or $V=\dot{x}$ for short). The metric $g$ on $M$ induces a metric $\hat{g}$ on $L M$ through

$$
\hat{g}_{x}\left(V_{1}, V_{2}\right)=\int_{0}^{1} d t g_{\mu \nu}(x(t)) V_{1}^{\mu}(x)(t) V_{2}^{\nu}(x)(t)
$$


Likewise, every $p$-form $\alpha$ on $M$ gives rise to a $p$-form $\hat{\alpha}$ on $L M$ via

$$
\hat{\alpha}_{x}\left(V_{1}, \ldots, V_{p}\right)=\int_{0}^{1} d t \alpha_{x(t)}\left(V_{1}(x)(t), \ldots, V_{p}(x)(t)\right),
$$

and a local basis of one-forms on $L M$ is given by the differentials $d x^{\mu}(t)$.

The last piece of information we need is that the Levi-Cività connection on $M$ can be pulled back to $S^{1}$ via a loop $x(t)$. This defines a covariant derivative on (3.2) and its dual which we denote by $\nabla_{t}$. We have e.g.

$$
\left(\nabla_{t} V^{\mu}\right)(x)(t)=\frac{d}{d t} V^{\mu}(x)(t)+\Gamma_{\nu \rho}^{\mu}(x(t)) \dot{x}^{\nu}(t) V^{\rho}(x)(t) .
$$

\subsection{Supersymmetric quantum mechanics}

We are now in a position to discuss example 1 of section 2.3 in more detail. In the notation of that section, we choose $X=L M, E=T X$, and $V=\dot{x}$. The anticommuting variables $\chi_{a}$ thus parametrize the fibres of $T X$ and we write them as $\chi_{a}=e_{a}^{\mu} \bar{\psi}_{\mu}$ where $e_{a}^{\mu}$ is the inverse vielbein corresponding to $g_{\mu \nu}$. Using the metric (3.3) as a fibre metric on $T_{x} X$, the first term of (2.28) is simply the standard bosonic kinetic term of quantum mechanics ,

$$
\xi^{2} / 2 \rightarrow \int_{0}^{\beta} d t g_{\mu \nu} \dot{x}^{\mu} \dot{x}^{\nu} / 2
$$

To put the remaining terms into a more familiar form, we use the standard trick of replacing the differentials $d x^{\mu}(t)$ by periodic anticommuting variables,

$$
d x^{\mu}(t) \rightarrow \psi^{\mu}(t)
$$

and integrating over them as well. As the integral over the $\psi$ 's will simply pick out the top-form part which is then to be integrated over $X$ (cf. (2.39)), nothing is changed by the substitution (3.7). With all this in mind the complete exponential of the Mathai-Quillen form $e_{V, \nabla}(L M)$ becomes

$$
S_{M}=\int_{0}^{\beta} d t\left[-g_{\mu \nu} \dot{x}^{\mu} \dot{x}^{\nu} / 2+R_{\rho \sigma}^{\mu \nu} \bar{\psi}_{\mu} \psi^{\rho} \bar{\psi}_{\nu} \psi^{\sigma} / 4-i \bar{\psi}_{\mu} \nabla_{t} \psi^{\mu}\right] .
$$

This is precisely the standard action of de Rham (or $N=1$ ) supersymmetric quantum mechanics to be found e.g. in [27, 28, 29, 30, 31] (with the spinors appearing there decomposed into their components; we also choose $\psi$ and $\bar{\psi}$ to be independent real fields instead of complex conjugates). It will be convenient to introduce a multiplier field $B_{\mu}$ and to rewrite the action (3.8) in first order form,

$$
S_{M}=\int_{0}^{\beta} d t\left[i \dot{x}^{\mu} B_{\mu}+g^{\mu \nu} B_{\mu} B_{\nu} / 2+R_{\rho \sigma}^{\mu \nu} \bar{\psi}_{\mu} \psi^{\rho} \bar{\psi}_{\nu} \psi^{\sigma} / 4-i \bar{\psi}_{\mu} \nabla_{t} \psi^{\mu}\right] .
$$


The supersymmetry of this action is

$$
\begin{aligned}
\delta x^{\mu} & =\psi^{\mu}, \quad \delta \bar{\psi}_{\mu}=B_{\mu}-\Gamma_{\mu \rho}^{\nu} \bar{\psi}_{\nu} \psi^{\rho} \\
\delta \psi^{\mu} & =0, \quad \delta B_{\mu}=\Gamma_{\mu \rho}^{\nu} B_{\nu} \psi^{\rho}-R_{\mu \rho \sigma}^{\nu} \bar{\psi}_{\nu} \psi^{\rho} \psi^{\sigma} / 2 .
\end{aligned}
$$

This is readily verified by noticing that $\delta^{2}=0$ and that (3.9) can itself be written as a supersymmetry variation,

$$
S_{M}=\delta \int_{0}^{\beta} d t\left[\bar{\psi}_{\mu}\left(i \dot{x}^{\mu}+g^{\mu \nu} B_{\nu} / 2\right)\right] .
$$

Note the similarity with (2.31). Reinterpreting $\delta$ as a BRST operator, this also shows that the sector of supersymmetric quantum mechanics annihilated by $\delta$ is topological (a BRST exact action being one of the hallmarks of topological field theory). As we will see below that only groundstates contribute to the partition function anyway, it is, in particular, independent of the coefficient of the second term of (3.11) regardless of whether we treat $\delta$ as a conventional supersymmetry operator (mapping bosonic to fermionic states and vice-versa) or as a BRST operator (annihilating physical states). Rescaling this term by a real parameter $\alpha$ we find the equivalent action

$$
S_{M}=\int_{0}^{\beta} d t\left[-g_{\mu \nu} \dot{x}^{\mu} \dot{x}^{\nu} / 2 \alpha+\alpha R_{\rho \sigma}^{\mu \nu} \bar{\psi}_{\mu} \psi^{\rho} \bar{\psi}_{\nu} \psi^{\sigma} / 4-i \bar{\psi}_{\mu} \nabla_{t} \psi^{\mu}\right] .
$$

On the other hand, if we rescale the time variable by $\beta$ we obtain the action (3.12) with $\int_{0}^{\beta} d t$ replaced by $\int_{0}^{1} d t$ and $\alpha$ replaced by $\beta$. Thus the 'topological' $\alpha$-independence translates into the quantum mechanical $\beta$-independence. Conversely, this $\beta$-independence is obvious from the standard Hamiltonian construction of supersymmetric quantum mechanics (cf. below) and translates into the topological $\alpha$-independence of (3.12).

This is not the place to enter into a detailed discussion of supersymmetric quantum mechanics, and we will in the following focus on those aspects relevant for the Mathai-Quillen side of the issue and our subsequent considerations involving topological gauge theories. For detailed discussions of supersymmetric quantum mechanics in the context of index theory and topological field theory the reader is referred to [30] and [9, pp. 140-176] respectively.

Our discussion of the Mathai-Quillen formalism suggests that the partition function $Z\left(S_{M}\right)$ of the supersymmetric quantum mechanics action $S_{M}$ (3.8), with periodic boundary conditions on all the fields, is the Euler number $\chi(M)$ of $M$ (as $\chi_{V}(L M)=\chi\left((L M)_{V}\right)=\chi(M)$, cf. (2.372.41)). As is well known, this is indeed the case.

The conventional way to see this (if one does not yet trust the infinite dimensional version of the Mathai-Quillen formalism) is to start with the definition of $\chi(M)$ as the Euler characteristic of $M(2.11)$. As there is a one-to-one correspondence between cohomology classes and harmonic forms on $M$ (more precisely, 
there is a unique representative in every de Rham cohomology class which is annihilated by the Laplacian $\left.\Delta=d d^{*}+d^{*} d\right)$ one can write $\chi(X)$ as a trace over the space $\operatorname{Ker} \Delta$,

$$
\chi(M)=\operatorname{tr}_{\text {Ker } \Delta}(-1)^{F},
$$

where $(-1)^{F}$ is $+1(-1)$ on even (odd) forms. As the operator $d+d^{*}$ commutes with $\Delta$ and maps even to odd forms and vice-versa, there is an exact pairing between 'bosonic' and 'fermionic' eigenvectors of $\Delta$ with non-zero eigenvalue. It is thus possible to extend the trace in (3.13) to a trace over the space of all differential forms,

$$
\chi(M)=\operatorname{tr}_{\Omega^{*}}(-1)^{F} e^{-\beta \Delta} .
$$

As only the zero modes of $\Delta$ will contribute to the trace, it is evidently independent of the value of $\beta$. Once one has put $\chi(M)$ into this form of a statistical mechanics partition function, one can use the Feynman-Kac formula to represent it as a supersymmetric path integral [30] with the action (3.8), imaginary time of period $\beta$ and periodic boundary conditions on the anticommuting variables $\psi^{\mu}$ (due to the insertion of $(-1)^{F}$ ). Conversely, a Hamiltonian analysis of the action (3.8) would tell us that we can represent its Hamiltonian by the Laplacian $\Delta$ on differential forms [28] and, tracing back the steps which led us to (3.14), we would then again deduce that $Z\left(S_{M}\right)=\chi(M)$, as anticipated in (2.40).

This Hamiltonian way of arriving at the action of supersymmetric quantum mechanics should be contrasted with the Mathai-Quillen approach. In the former one starts with the operator whose index one wishes to calculate (e.g. $\left.d+d^{*}\right)$, constructs a corresponding Hamiltonian, and then deduces the action. On the other hand, in the latter one begins with a finite dimensional topological invariant (e.g. $\chi(M))$ and represents that directly as an infinite dimensional integral, the partition function of a supersymmetric action.

What makes such a path integral representation of $\chi(M)$ interesting is that one can now go ahead and try to somehow evaluate it directly, thus possibly obtaining alternative expressions for $\chi(M)$. Indeed, one can obtain path integral 'proofs' of the Gauss-Bonnet and Poincaré-Hopf theorems in this way. This is just the infinite dimensional analogue of the considerations of section 2.2 where different choices of $s$ in $\int_{X} e_{s, \nabla}(E)$ lead to different expressions for $\chi(E)$. As we will derive the finite dimensional Mathai-Quillen form from supersymmetric quantum mechanics in section 3.3 we can appeal to the manipulations of section 2.2 to complete these 'proofs'. However, it is also instructive to perform these calculations directly. Before indicating how this can be done, we will need to introduce a generalization of the action (3.8) which arises when one takes the section $\dot{x}^{\mu}+\gamma g^{\mu \nu} \partial_{\nu} W$ of $T(L M)$ (cf. example 1 of section 2.3) to regularize the Euler number of $L M$. Here $W$ is a function (potential) on $M$ and $\gamma$ is yet one more arbitrary real parameter. In that case one obtains (introducing also the 
parameter $\alpha$ of $(3.12))$

$$
\begin{aligned}
S_{M, \gamma W}= & \int_{0}^{\beta} d t\left[i\left(\dot{x}^{\mu}+\gamma g^{\mu \nu} \partial_{\nu} W(x)\right) B_{\mu}+\alpha g^{\mu \nu} B_{\mu} B_{\nu} / 2+\alpha R_{\rho \sigma}^{\mu \nu} \bar{\psi}_{\mu} \psi^{\rho} \bar{\psi}_{\nu} \psi^{\sigma} / 4\right. \\
& \left.-i \bar{\psi}_{\mu}\left(\delta_{\nu}^{\mu} \nabla_{t}+\gamma g^{\mu \rho} \nabla_{\rho} \partial_{\nu} W\right) \psi^{\nu}\right] .
\end{aligned}
$$

From the Hamiltonian point of view this action arises from replacing the exterior derivative $d$ by

$$
d \rightarrow d_{\gamma W} \equiv e^{-\gamma W} d e^{\gamma W}
$$

and applying the above procedure to the corresponding Laplacian $\Delta_{\gamma W}$. As there is a one-to-one correspondence between $\Delta$ - and $\Delta_{\gamma W}$-harmonic forms, this also represents $\chi(M)$ (independently of the value of $\gamma$ ).

This freedom in the choice of parameters $\alpha, \beta, \gamma$ greatly facilitates the evaluation of the partition function. Let us, for example, choose $\alpha=0$ in (3.15). Then the curvature term drops out completely and the $B$-integral will simply give us a delta function constraint $\dot{x}^{\mu}+\gamma g^{\mu \nu} \partial_{\nu} W=0$. Squaring this equation and integrating it over $t$ one finds

$$
\begin{aligned}
& \dot{x}^{\mu}+\gamma g^{\mu \nu} \partial_{\nu} W=0 \\
\rightarrow & \int_{0}^{\beta} d t g_{\mu \nu} \dot{x}^{\mu} \dot{x}^{\nu}+\gamma^{2} g^{\mu \nu} \partial_{\mu} W \partial_{\nu} W+2 \gamma \dot{x}^{\mu} \partial_{\mu} W=0 \\
\rightarrow & \dot{x}^{\mu}=0=\partial_{\mu} W
\end{aligned}
$$

as the second line is the sum of two nonnegative terms and a total derivative. This is the 'squaring argument' referred to in section 2.3. It demonstrates that the path integral over $L M$ is reduced to an integral over $M$ (by $\dot{x}^{\mu}=0$ ) and further to an integral over the set $M_{W^{\prime}}$ of critical points of $W$ (and analogously for the $\psi$ 's by supersymmetry). When the critical points are isolated, inspection of (3.15) immediately reveals that the partition function is

$$
\chi(M)=Z\left(S_{M, W}\right)=\sum_{x_{k}: d W\left(x_{k}=0\right.} \operatorname{sign}\left(\operatorname{det} H_{x_{k}}(W)\right),
$$

where

$$
H_{x_{k}}(W)=\left(\nabla_{\mu} \partial_{\nu} W\right)\left(x_{k}\right)
$$

is the Hessian of $W$ at $x_{k}$. This is the Poincaré-Hopf theorem (2.9). This result can also be derived by keeping $\alpha$ non-zero and taking the limit $\gamma \rightarrow \infty$ instead which also has the effect of localizing the path integral around the critical points of $W$ because of the term $\gamma^{2} W^{2}$ in the action.

If we switch off the potential, then we can not simply set $\alpha=0$ in (3.15), as the resulting path integral would be singular due to the undamped bosonic and fermionic zero modes. In that case, the limit $\alpha \rightarrow 0$ or $\beta \rightarrow 0$ has to be taken with more care. Since whatever we can do with $\alpha$ we can also do with $\beta$ let us set 
$\alpha=1$ in the following. We first rescale the time coordinate $t$ by $\beta$, and then we rescale $B$ and $\bar{\psi}$ by $\beta^{1 / 2}, B \rightarrow \beta^{1 / 2} B$ and $\bar{\psi} \rightarrow \beta^{1 / 2} \bar{\psi}$, and all the non-zero-modes of $x$ and $\psi$ by $\beta^{-1 / 2}$. This will leave the path integral measure invariant and has the effect that all the $\beta$-dependent terms in the action are at least of order $O\left(\beta^{1 / 2}\right)$ and the limit $\beta \rightarrow 0$ can now be taken with impunity. The integral over the non-constant modes gives 1 and the net-effect of this is that one is left with a finite-dimensional integral of the form (2.25), namely

$$
\chi(M) \sim \int d x \int d \psi \int d \bar{\psi} e^{R_{\rho \sigma}^{\mu \nu} \bar{\psi}_{\mu} \psi^{\rho} \bar{\psi}_{\nu} \psi^{\sigma} / 4}
$$

over the constant modes of $x, \psi$, and $\bar{\psi}$ of which there are $\operatorname{dim}(M)$ each. In order to get a non-zero contribution (i.e. to soak up the fermionic zero modes) one has to expand (3.20) to $(\operatorname{dim}(M) / 2)$ 'th order, yielding the Pfaffian of $\mathcal{R}_{M}$ and hence, upon integration over $M$ (the $x$ zero modes) the Gauss-Bonnet theorem (2.6, 2.10). (3.20) also gives the correct result for odd dimensional manifolds, $\chi(M)=0$, as there is no way to pull down an odd number of $\psi$ 's and $\bar{\psi}$ 's from the exponent.

If the critical points of $W$ are not isolated then, by a combination of the above arguments, one recovers the generalization $\chi(M)=\chi\left(M_{W^{\prime}}\right)(2.12,2.43)$ of the Poincaré-Hopf theorem in the form (2.35).

As this treatment of supersymmetric quantum mechanics has admittedly been somewhat sketchy I should perhaps, summarizing this section, state clearly what are the important points to keep in mind:

1. The Mathai-Quillen formalism applied to the loop space $L M$ of a Riemannian manifold $M$ leads directly to the action of supersymmetric quantum mechanics with target space $M$. Different sections lead to different actions, and those we have considered all regularize the Euler number of $L M$ to be $\chi(M)$.

2. Explicit evaluation of the supersymmetric quantum mechanics path integrals obtained in this way confirms that we can indeed represent the regularized Euler number $\chi_{V}(L M)$, as defined by (2.37), by the functional integral (2.38).

3. Finally, I have argued (although not proved in detail) that the zero modes are all that matter in supersymmetric quantum mechanics, the integral over the non-zero-modes giving 1 . This observation is useful when one attempts to construct topological gauge theories from supersymmetric quantum mechanics on spaces of connections (see [18] and the remarks in section $4.3)$. 


\subsection{The Mathai-Quillen form from supersymmetric quan- tum mechanics}

So far we have derived the action of supersymmetric quantum mechanics by formally applying the Mathai-Quillen formalism to $L M$, and we have indicated how to rederive the classical (generalized) Poincaré-Hopf and Gauss-Bonnet formulae. What is still lacking to complete the picture is a derivation of the general (finite dimensional) Mathai-Quillen form $\Phi_{\nabla}(E)$ (2.27) for $E=T M$ from supersymmetric quantum mechanics.

As $\Phi_{\nabla}(T M)$ can be pulled back to $M$ via an arbitrary vector field (section of $T M) v$, not necessarily a gradient vector field, we need to consider the supersymmetric quantum mechanics action resulting from the regularizing section $\dot{x}+\gamma v$ of $T(L M)$. This is just the action (3.15) with $\partial_{\nu} W$ replaced by $g_{\mu \nu} v^{\mu}$. In that case the squaring argument, as expressed in (3.17), fails because the cross-term will not integrate to zero. In the limit $\gamma \rightarrow \infty$ the path integral will nevertheless reduce to a Gaussian around the zero locus of $v$ because of the term $\gamma^{2} g_{\mu \nu} v^{\mu} v^{\nu}$ in the action, and in this limit the path integral calculates $\chi(M)=\chi\left(M_{v}\right)$ in the form (2.35).

To derive the Mathai-Quillen form, however, we are interested in finite values of $\gamma$. Thus, what we need to do now is adjust the parameters in such a way that the zero modes of all the terms involving the vector field $v$ or the curvature survive. Proceeding exactly as in the derivation of the Gauss-Bonnet theorem one ends up with a time-independent 'action' of the form

$$
B^{2} / 2+\gamma v^{\mu} B_{\mu}+R_{\rho \sigma}^{\mu \nu} \bar{\psi}_{\mu} \bar{\psi}_{\nu} \psi^{\rho} \psi^{\sigma} / 4-i \gamma \bar{\psi}_{\mu} \nabla_{\nu} v^{\mu} \psi^{\nu}
$$

which - upon integration over $B$ - reproduces precisely the exponent (2.28) of the Mathai-Quillen form (2.27) with $\xi^{a}$ replaced by the arbitrary section $\gamma v^{\mu}$ or $\gamma e_{\mu}^{a} v^{\mu}$ of $T M$. We have thus also rederived the Mathai-Quillen formula (2.13) for $T M$,

$$
\chi(M)=\int_{M} e_{v, \nabla}(T M),
$$

from supersymmetric quantum mechanics. Specializing now to $v=0$ or $v$ a generic vector field with isolated zeros again reproduces the classical expressions.

\section{The Euler Number of Vector Bundles over $\mathcal{A} / \mathcal{G}$ and Topological Gauge Theory}

In this section we essentially work out the details of examples 2 and 3 and discuss some related models as well. Section 4.1 contains a brief summary of the facts we will need from the geometry of gauge theories. In section 4.2 we will see how Donaldson theory can be interpreted in terms of the Mathai-Quillen formalism. Section 4.3 sketches the construction of a topological gauge theory in $3 d$ from 
the tangent bundle over or (alternatively) supersymmetric quantum mechanics on gauge orbit space which represents the Euler characteristic of the moduli space of flat connections. It also contains a brief discussion of the $2 d$ analogue of Donaldson theory.

\subsection{Geometry of gauge theories}

Let $(M, g)$ be a compact, oriented, Riemannian manifold, $\pi: P \rightarrow M$ a principal $G$ bundle over $M, G$ a compact semisimple Lie group and $\mathbf{g}$ its Lie algebra. We denote by $\mathcal{A}$ the space of (irreducible) connections on $P$, and by $\mathcal{G}$ the infinite dimensional gauge group of vertical automorphisms of $P$ (modulo the center of $G)$. Then $\mathcal{G}$ acts freely on $\mathcal{A}$ and

$$
\Pi: \mathcal{A} \rightarrow \mathcal{A} / \mathcal{G}
$$

is a principal $\mathcal{G}$ bundle. The aim of this section will be to determine a connection and curvature on this principal bundle, so that we can write down (or recognize) the Mathai-Quillen form for some infinite dimensional vector bundles associated to it. We will also state the Gauss-Codazzi equation which express the Riemann curvature tensor $\mathcal{R}_{\mathcal{M}}$ of some moduli subspace $\mathcal{M}$ of $\mathcal{A} / \mathcal{G}$ in terms of the curvature of $\mathcal{A} / \mathcal{G}$ and the extrinsic curvature (second fundamental form) of the embedding $\mathcal{M} \hookrightarrow \mathcal{A} / \mathcal{G}$. The details can be found e.g. in [32, 33, 34, 35].

Continuing with notation, we denote by $\Omega^{k}(M, \mathbf{g})$ the space of $k$-forms on $M$ with values in the adjoint bundle $a d P:=P \times_{a d} \mathbf{g}$ and by

$$
d_{A}: \Omega^{k}(M, \mathbf{g}) \rightarrow \Omega^{k+1}(M, \mathbf{g})
$$

the covariant exterior derivative with curvature $\left(d_{A}\right)^{2}=F_{A}$. The spaces $\Omega^{k}(M, \mathbf{g})$ have natural scalar products defined by the metric $g$ on $M$ (and the corresponding Hodge operator $*$ ) and an invariant scalar product $t r$ on $\mathbf{g}$, namely

$$
\langle X, Y\rangle=\int_{M} \operatorname{tr}(X * Y), \quad X, Y \in \Omega^{k}(M, \mathbf{g})
$$

(I hope that occasionally denoting these forms by $X$ as well will not give rise to any confusion with the manifold $X$ of section 2). The tangent space $T_{A} \mathcal{A}$ to $\mathcal{A}$ at a connection $A$ can be identified with $\Omega^{1}(M, \mathbf{g}$ ) (as $\mathcal{A}$ is an affine space, two connections differing by an element of $\Omega^{1}(M, \mathbf{g})$ ). Equation (4.3) thus defines a metric $g_{\mathcal{A}}$ on $\mathcal{A}$. The Lie algebra of $\mathcal{G}$ can be identified with $\Omega^{0}(M, \mathbf{g})$ and acts on $A \in \mathcal{A}$ via gauge transformations,

$$
A \mapsto A+d_{A} \Lambda, \quad \Lambda \in \Omega^{0}(M, \mathbf{g}),
$$

so that $d_{A} \Lambda$ is the fundamental vector field at $A$ corresponding to $\Lambda$. At each point $A \in \mathcal{A}, T_{A} \mathcal{A}$ can thus be split into a vertical part $V_{A}=\operatorname{Im}\left(d_{A}\right)$ (tangent 
to the orbit of $\mathcal{G}$ through $A$ ) and a horizontal part $H_{A}=\operatorname{Ker}\left(d_{A}^{*}\right)$ (the orthogonal complement of $V_{A}$ with respect to the scalar product (4.3)). Explicitly this decomposition of $X \in \Omega^{1}(M, \mathbf{g})$ into its vertical and horizontal parts is

$$
\begin{aligned}
X & =d_{A} G_{A}^{0} d_{A}^{*} X+\left(X-d_{A} G_{A}^{0} d_{A}^{*} X\right) \\
& \equiv v_{A} X+h_{A} X
\end{aligned}
$$

where $G_{A}^{0}=\left(d_{A}^{*} d_{A}\right)^{-1}$ is the Greens function of the scalar Laplacian (which exists if $A$ is irreducible). We will identify the tangent space $T_{[A]} \mathcal{A} / \mathcal{G}$ with $H_{A}$ for some representative $A$ of the gauge equivalence class $[A]$.

Then $g_{\mathcal{A}}$ induces a metric $g_{\mathcal{A} / \mathcal{G}}$ on $\mathcal{A} / \mathcal{G}$ via

$$
g_{\mathcal{A} / \mathcal{G}}([X],[Y])=g_{\mathcal{A}}\left(h_{A} X, h_{A} Y\right),
$$

where $X, Y \in \Omega^{1}(N, \mathbf{g})$ project to $[X],[Y] \in T_{[A]} \mathcal{A} / \mathcal{G}$. With the same notation the Riemannian curvature of $\mathcal{A} / \mathcal{G}$ is

$$
\begin{aligned}
\left\langle\mathcal{R}_{\mathcal{A} / \mathcal{G}}([X],[Y])[Z],[W]\right\rangle & =\left\langle *\left[h_{A} X, * h_{A} W\right], G_{A}^{0} *\left[h_{A} Y, * h_{A} Z\right]\right\rangle-(X \leftrightarrow Y) \\
& +2\left\langle *\left[h_{A} W, * h_{A} Z\right], G_{A}^{0} *\left[h_{A} X, * h_{A} Y\right]\right\rangle
\end{aligned}
$$

If $\mathcal{M}$ is some embedded submanifold of $\mathcal{A} / \mathcal{G}$, then (4.6) induces a metric $g_{\mathcal{M}}$ on $\mathcal{M}$ whose Riemann curvature tensor is

$$
\begin{aligned}
\left\langle\mathcal{R}_{\mathcal{M}}([X],[Y])[Z],[W]\right\rangle & =\left\langle\mathcal{R}_{\mathcal{A} / \mathcal{G}}([X],[Y])[Z],[W]\right\rangle \\
& +\left(\left\langle K_{\mathcal{M}}([Y],[Z]), K_{\mathcal{M}}([X],[W])\right\rangle-(X \leftrightarrow Y)\right)
\end{aligned}
$$

where $K_{\mathcal{M}}$ is the extrinsic curvature (or second fundamental form) of $\mathcal{M}$ in $\mathcal{A} / \mathcal{G}$. For instanton moduli spaces $K_{\mathcal{M}}$ has been computed in [35] and for moduli spaces of flat connections in two and three dimensions one finds [17]

$$
K_{\mathcal{M}}([X],[Y])=-d_{A}^{*} G_{A}^{2}\left[\bar{X}_{A}, \bar{Y}_{A}\right]
$$

Here the tangent vectors $[X]$ and $[Y]$ to $\mathcal{M}$ are represented on the right hand side by elements $\bar{X}$ and $\bar{Y}$ of $\Omega^{1}(M, \mathbf{g})$ satisfying both the horizontality condition $d_{A}^{*} \bar{X}=d_{A}^{*} \bar{Y}=0$ and the linearized flatness equation $d_{A} \bar{X}=d_{A} \bar{Y}=0 . G_{A}^{2}$ is the Greens function of the Laplacian on two-forms and in the three-dimensional case we think of it as being composed with a projector onto the orthogonal complement of the zero modes of the Laplacian. Thus

$$
\left\langle K_{\mathcal{M}}([Y],[Z]), K_{\mathcal{M}}([X],[W])\right\rangle=\left\langle\left[\bar{Y}_{A}, \bar{Z}_{A}\right], G_{A}^{2}\left[\bar{X}_{A}, \bar{W}_{A}\right]\right\rangle
$$

and together with (4.7) and (4.8) this determines $\mathcal{R}_{\mathcal{M}}$ entirely in terms of Greens functions of differential operators on $M$. It is in this form that we will encounter $\mathcal{R}_{\mathcal{M}}$ in section 4.3 . 
The decomposition (4.5) also defines a connection on the principal bundle $\mathcal{A} \rightarrow \mathcal{A} / \mathcal{G}$ itself, with connection form $\theta_{\mathcal{A}}=G_{A}^{0} d_{A}^{*}$. Indeed, $\theta_{\mathcal{A}}$ can be regarded as a Lie algebra $\left(=\Omega^{0}(M, \mathbf{g})\right)$ valued one-form on $\mathcal{A}$,

$$
\begin{aligned}
\theta_{\mathcal{A}}: T_{A} \mathcal{A} & \rightarrow \Omega^{0}(M, \mathbf{g}) \\
X & \mapsto \theta_{\mathcal{A}}(X)=G_{A}^{0} d_{A}^{*} X .
\end{aligned}
$$

It transforms homogenously under gauge transformations, is obviously vertical (i.e. vanishes on $\operatorname{Ker}\left(d_{A}^{*}\right)$ ), and assigns to the fundamental vector field $d_{A} \Lambda$ the corresponding Lie algebra element

$$
\theta_{\mathcal{A}}\left(d_{A} \Lambda\right)=G_{A}^{0} d_{A}^{*} d_{A} \Lambda=\Lambda,
$$

as behoves a connection form. Its curvature is the horizontal two-form

$$
\Theta_{\mathcal{A}}=d_{\mathcal{A}} \theta_{\mathcal{A}}+\frac{1}{2}\left[\theta_{\mathcal{A}}, \theta_{\mathcal{A}}\right]
$$

$\left(d_{\mathcal{A}}\right.$ denotes the exterior derivative on $\left.\mathcal{A}\right)$. Evaluated on horizontal vectors $X, Y \in$ $H_{A}$ the second term is zero and from the first term only the variation of $A$ in $d_{A}^{*}$ will contribute (because otherwise the surviving $d_{A}^{*}$ will annihilate either $X$ or $Y)$. Thus one finds

$$
\Theta_{\mathcal{A}}(X, Y)=G_{A}^{0} *[X, * Y],
$$

a formula that we will reencounter in our discussion of Donaldson theory below.

Finally, we will introduce the bundles $\mathcal{E}_{0}$ and $\mathcal{E}_{+}$which will play a role in the interpretation of topological gauge theories from the Mathai-Quillen point of view below. If $\operatorname{dim}(M)=2$, we consider the bundle

$$
\mathcal{E}_{0}:=\mathcal{A} \times{ }_{\mathcal{G}} \Omega^{0}(M, \mathbf{g})
$$

associated to the principal bundle (4.1) via the adjoint representation. If $\operatorname{dim}(M)=$ 4 , we choose as fibre the space $\Omega_{+}^{2}(M, \mathbf{g})$ of self-dual two-forms. One then has the associated vector bundle

$$
\mathcal{E}_{+}:=\mathcal{A} \times_{\mathcal{G}} \Omega_{+}^{2}(M, \mathbf{g})
$$

over $\mathcal{A} / \mathcal{G}$. In the standard manner (4.15) and (4.16) inherit the connection (4.11) and its curvature (4.14) from the parent principal bundle $\mathcal{A} \rightarrow \mathcal{A} / \mathcal{G}$ (4.1).

\subsection{The Atiyah-Jeffrey Interpretation of Donaldson the- ory}

Donaldson theory [1] is the prime example of a cohomological field theory. It was introduced by Witten to give a field theoretic description of the intersection 
numbers of moduli spaces of instantons investigated by Donaldson [12]. Donaldson's introduction of gauge theoretic methods into the study of four-manifolds has had enormous impact on the subject (see [36] for reviews), but unfortunately it would require a seperate set of lectures to describe at least the basic ideas. Likewise, it is not possible to give an account of the field theoretic description here which would do justice to the many things that can and should be said about Donaldson theory. Therefore, I will make only a few general remarks on the structure of the action of Donaldson theory and other cohomological field theories describing intersection theory on moduli spaces. The main aim of this section will, of course, be to show that this action is, despite appearance, also of the Mathai-Quillen type. For a review of both the mathematical and the physical side of the story see [9, pp. 198-247].

The action of Donaldson theory on a four-manifold $M$ in equivariant form (i.e. prior to the introduction of gauge ghosts) is [1]

$$
\begin{aligned}
S_{D}= & \int_{M}\left(B_{+}\left(F_{A}\right)_{+}+\chi_{+}\left(d_{A} \psi\right)_{+}-\alpha B_{+}^{2} / 2+\eta d_{A} * \psi\right) \\
& +\left(\bar{\phi} d_{A} * d_{A} \phi+\bar{\phi}[\psi, * \psi]-\alpha \phi\left[\chi_{+}, \chi_{+}\right] / 2\right) .
\end{aligned}
$$

Here $(.)_{+}$denotes projection onto the self-dual part of a two-form,

$$
\left(F_{A}\right)_{+}=\frac{1}{2}\left(F_{A}+* F_{A}\right), \quad *\left(F_{A}\right)_{+}=\left(F_{A}\right)_{+},
$$

etc. Furthermore $\psi \in \Omega^{1}(M, \mathbf{g})$ is a Grassmann odd Lie algebra valued one-form with ghost number 1 . It is (as in supersymmetric quantum mechanics) the superpartner of the fundamental bosonic variable $A$ and represents tangent vectors to $\mathcal{A}$. $\left(B_{+}, \chi_{+}\right)$are self-dual two-forms with ghost numbers $(0,-1)$ (Grassmann parity (even,odd)), and $(\phi, \bar{\phi}, \eta)$ are elements of $\Omega^{0}(M, \mathbf{g})$ with ghost numbers $(2,-2,-1)$ and parity (even,even,odd). $\alpha$ is a real parameter whose significance is the same as that played by $\alpha$ in supersymmetric quantum mechanics (cf. (3.15)). This action has an equivariantly nilpotent BRST-like symmetry

$$
\begin{aligned}
\delta A & =\psi & \delta \psi & =-d_{A} \phi \\
\delta \chi_{+} & =B_{+} & \delta B_{+} & =\left[\phi, \chi_{+}\right] \\
\delta \bar{\phi} & =\eta & \delta \eta & =[\phi, \bar{\phi}] \\
\delta \phi & =0 & \delta^{2} & =\delta_{\phi}
\end{aligned}
$$

where $\delta_{\phi}$ denotes a gauge variation with respect to $\phi$. From these transformations it can be seen that the action $S_{D}$ is BRST-exact,

$$
S_{D}=\delta \int_{M} \chi_{+}\left(\left(F_{A}\right)_{+}-\alpha B_{+} / 2\right)+\bar{\phi} d_{A} * \psi .
$$

(cf. (2.31,3.11)). The single most important consequence of (4.20), which we will abbreviate to $S_{D}=\delta \Sigma_{D}$, is that the partition function $Z\left(S_{D}\right)$ of $S_{D}$ is given 
exactly by its one-loop approximation. Likewise, it is independent of the metric on $M$ and any other 'coupling constants' which may enter into its construction in addition to $\hbar$ and $g_{\mu \nu}$. E.g. for the metric the argument runs as follows. Although $g_{\mu \nu}$ enters in a number of places in (4.17), a variation of it produces an insertion of a BRST-exact operator into the path integral whose vacuum expectation value vanishes provided that the vacuum is BRST invariant,

$$
\begin{aligned}
\frac{\delta}{\delta g_{\mu \nu}} Z\left(S_{D}\right) & =\frac{\delta}{\delta g_{\mu \nu}} \int e^{-\delta \Sigma_{D}} \\
& =-\left\langle 0\left|\delta\left(\frac{\delta}{\delta g_{\mu \nu}} \Sigma_{D}\right)\right| 0\right\rangle=0 .
\end{aligned}
$$

By the same argument, $Z\left(S_{D}\right)$ is independent of $\alpha$ and correlation functions of metric independent and BRST invariant operators are themselves metric independent. We will briefly come back to these 'observables' of Donaldson theory below.

Equation (4.20) also makes the significance of the individual terms in (4.17) more transparent. In particular, one sees that the first term of (4.20) imposes a delta function $(\alpha=0)$ or Gaussian (for $\alpha \neq 0$ ) constraint onto the instanton configurations $\left(F_{A}\right)_{+}=0$. Together with the gauge fixing of the gauge fields $A$, implicit in the above, this localizes the path integral around the instanton moduli space $\mathcal{M}_{I}$. The second term, on the other hand, fixes the tangent vector $\psi$ to be horizontal, i.e. to satisfy $d_{A}^{*} \psi=0$, and $\psi$ thus represents a tangent vector to $\mathcal{A} / \mathcal{G}$. Moreover, the $\chi_{+}$equation of motion restricts $\psi$ further to be tangent to $\mathcal{M}_{I}$, i.e. to satisfy the linearized instanton equation $\left(d_{A} \psi\right)_{+}=0$ (modulo irrelevant terms proportional to $\alpha$ ). The number of $\psi$ zero modes will thus (generically, see [1], 9]) be equal to the dimension $d(\mathcal{M})$ of $\mathcal{M}_{I}$.

The structure of Donaldson theory summarized in the preceding paragraphs is prototypical for the actions of cohomological field theories in general: Given the moduli space $\mathcal{M}$ of interest, one seeks a description of it in terms of certain fields (e.g. connections), field equations (e.g. $\left(F_{A}\right)_{+}=0$ ), and their symmetries (e.g. gauge symmetries). One then constructs an action which is essentially a bunch of delta functions or Gaussians around the desired field configurations and (by supersymmetry) their tangents. Thus, a topological action describing intersection theory on the moduli space of flat connections on some $n$-manifold $M$ would roughly be of the form

$$
\left.S \sim \int_{M} B_{n-2} F_{A}+(\text { super partners })+\text { (gauge fixing terms }\right),
$$

where $B \in \Omega^{n-2}(M, \mathbf{g})$ and (for the cognoscenti) 'gauge fixing terms' is meant to also include all the terms corresponding to the higher cohomology groups of the deformation complex of $\mathcal{M}$, i.e. to the tower of Bianchi symmetries $\delta_{B} B_{n-2}=$ $d_{A} B_{n-3}, \delta_{B} B_{n-3}=\ldots$.

Evidently, this is quite a pragmatic and not very sophisticated way of looking at topological field theory. It will, however, be good enough for the time being. 
Later on we will see how to construct the action (4.22) from the more satisfactory Mathai-Quillen point of view. For an elaboration of the axiomatic approach initiated by Atiyah [37] see [38, chs. 3 and 4].

Let us now return to Donaldson theory and show that its action $S_{D}$ is of the Mathai-Quillen form. We will do this by making use of the equations of motion arising from (4.20) (which is legitimate since all the integrals are Gaussian). We set $\alpha=1$ in the following.

- Integrating out $B$ one obtains the term $-\left(F_{A}\right)_{+}^{2} / 2$

- The $\eta$-equation implies that $\psi$ is horizontal which is henceforth tacitly understood

- The $\bar{\phi}$ equation of motion yields

$$
\phi=G_{A}^{0} *[\psi, * \psi],
$$

and, plugged back into the action, this gives rise to the term

$$
-\left[\chi_{+}, \chi_{+}\right] G_{A}^{0}[\psi, * \psi] / 2
$$

- Putting all this together we see that effectively the action of Donaldson theory is

$$
S_{D}=\int_{M}-\left(F_{A}\right)_{+}^{2} / 2-\left[\chi_{+}, \chi_{+}\right] G_{A}^{0}[\psi, * \psi] / 2+\left(d_{A} \psi\right)_{+} \chi_{+} .
$$

Let us now compare this with (2.28). We see that, apart from a factor of $i$ which is not terribly important and which can be smuggled back into (4.17) and (4.24) by appropriate scaling of the fields), the correspondence is perfect. From the identification $\chi_{a} \sim \chi_{+}$we read off that the standard fibre of the sought for vector bundle is $\Omega_{+}^{2}(M, \mathbf{g})$. The section is obviously $s(A)=\left(F_{A}\right)_{+}$, and as this transforms in the adjoint under gauge transformations the vector bundle in question has to be the bundle $\mathcal{E}_{+}$introduced in (4.16). This is also confirmed by a comparison of the second term of (2.28) with the second term of (4.24) and the curvature form $\Theta_{\mathcal{A}}$ (4.14). Thus we finally arrive at the desired equation [11]

$$
Z\left(S_{D}\right)=\chi_{s}\left(\mathcal{E}_{+}\right)
$$

identifying the partition function of Donaldson theory as the regularized Euler number of the infinite dimensional vector bundle $\mathcal{E}_{+}$and proving the result claimed in example 2 of section 2.3.

One important point we have ignored so far is that the partition function $Z\left(S_{D}\right)$ will be zero whenever there are $\psi$ zero modes, i.e. whenever the dimension $d(\mathcal{M})$ of $\mathcal{M}_{I}$ is non-zero. This is in marked contrast with the situation 
we encountered in supersymmetric quantum mechanics in section 3 . There the partition function $Z\left(S_{M}\right)=\chi(M)$ was generally non-zero, despite the presence of $\operatorname{dim}(M) \psi$ zero modes. I will now briefly try to explain the reason for this difference and the related issue of observables in Donaldson theory (with no claim to completeness nor to complete comprehensibility):

In supersymmetric quantum mechanics there are an equal number of $\psi$ and $\bar{\psi}$ zero modes, and these can be soaked up by expanding the curvature term (which contains an equal number of $\psi$ 's and $\bar{\psi}$ 's) to the appropriate power. In Donaldson theory the role of $\bar{\psi}$ is played by $\chi_{+}$. Generically, however, there will be no $\chi_{+}$zero modes at all, independently of the dimension of the moduli space, so that the fermionic $\psi$ zero modes can not be soaked up by the curvature term of (4.24). (As an aside: the $\chi_{+}$zero modes represent the second cohomology group of the instanton deformation complex and thus, together with reducible connections, the obstruction to having a smooth moduli space. For the class of four-manifolds considered in [12] it can be shown that this cohomology group is zero at irreducible instantons for a generic metric.)

Thus, in order to get a non-zero result one has to insert operators into the path integral which take care of the $\psi$ zero modes or, in other words, one has to construct a top-form on $\mathcal{M}_{I}$ which can then be integrated over it. These operators have to be BRST invariant, and - in view of (4.19) - this translates into the requirement that they represent cohomology classes of $\mathcal{A} / \mathcal{G}$. This is just like the situation we considered at the end of section 2. When there is a mismatch between the rank $2 m$ of $E$ and the dimension $n$ of $X$ one can obtain non-zero numbers by pairing $e_{\nabla}(E)$ with representatives of $H^{n-2 m}(X)$. Likewise, even if $n=2 m$ but one chooses a non-generic section of $E$ with a $k$-dimensional zero locus, this can be represented by an $(n-k)$-form which still has to be paired with a $k$-form in order to make it a volume form on $X$. In the case of Donaldson theory we have chosen a section with a $d(\mathcal{M})$-dimensional zero locus and we have to pair the corresponding Euler class, the integrand of (4.25), with $d(\mathcal{M})$-forms on $\mathcal{A} / \mathcal{G}$ to produce a good volume form on $\mathcal{A} / \mathcal{G}$ which will then localize to a volume form on $\mathcal{M}_{I}$. In the work of Donaldson the cohomology classes considered for this purpose are certain characteristic classes (of the universal bundle of [39]) which also arise naturally in the field theoretic description [40, 9]. For instance, one of the building blocks is the two-form $\phi$ as given by (4.23) which represents the curvature form $\Theta_{\mathcal{A}}$ (4.14). Unfortunately, these intersection numbers are very difficult to calculate in general. For details please consult the cited literature.

\subsection{Flat connections in two and three dimensions}

It is, of course, also possible to turn around the strategy of the previous section, i.e. to start with the Mathai-Quillen formalism applied to some vector bundle over $\mathcal{A} / \mathcal{G}$ and to then reconstruct the action of the corresponding topological gauge theory from there. 
Let us, for instance, consider the problem of constructing a topological gauge theory in $3 d$ whose partition function (formally) calculates the Euler characteristic $\chi\left(\mathcal{M}^{3}\right)$ of the moduli space $\mathcal{M}^{3}=\mathcal{M}^{3}(M, G)$ of flat $G$ connections on some three-manifold $M$. We actually already know two ways of achieving this, provided that we can find a vector field $v$ on $\mathcal{A}^{3} / \mathcal{G}^{3}$ (the superscripts are a reminder of the dimension we are in) whose zero locus is $\mathcal{M}^{3}$. Fortuitously, in three dimensions such a vector field exists, namely $v=* F_{A}$. A priori, this only defines a vector field on $\mathcal{A}^{3}$, as $* F_{A} \in \Omega^{1}(M, \mathbf{g})$. It is, however, horizontal $\left(d_{A}^{*} * F_{A}=0\right.$ by the Bianchi identity $\left.d_{A} F_{A}=0\right)$ and thus projects to a vector field on $\mathcal{A}^{3} / \mathcal{G}^{3}$ whose zero locus is $\mathcal{M}^{3}$. This vector field is the gradient vector field of the Chern-Simons functional

$$
C S(A)=\int_{M} A d A+\frac{2}{3} A^{3}
$$

whose critical points are well known to be the flat connections. (Of course, this does not really define a functional on $\mathcal{A}^{3} / \mathcal{G}^{3}$, as it changes by a constant times the winding number under large gauge transformations. But its derivative is well defined and this non-invariance implies that the one-form $d_{\mathcal{A}} C S(A)$ passes down to a closed but not exact one-form $\mathcal{F}_{A}$ on $\mathcal{A}^{3} / \mathcal{G}^{3}$. Explicitly, $\mathcal{F}_{A}$ is given by

$$
\begin{aligned}
\mathcal{F}_{A}: T_{[A]} \mathcal{A}^{3} / \mathcal{G}^{3} & \rightarrow \mathbf{R} \\
{[X] } & \rightarrow \int_{M} F_{A} X .
\end{aligned}
$$

Note that this does not depend on the representative of $[X]$ as $\int_{M} F_{A} d_{A} \Lambda=0$.) In two dimensions such a vector field appears not to exist at first sight and one has to be a little more inventive (cf. [17] and the remarks at the end of this section).

Given this vector field, the first possibility is then to adapt the Atiyah-Jeffrey construction of the previous section to the case $X=\mathcal{A}^{3} / \mathcal{G}^{3}$ and $E=T\left(\mathcal{A}^{3} / \mathcal{G}^{3}\right)$, to use $v=* F_{A}$ as the regularizing section for

$$
\chi_{v}\left(\mathcal{A}^{3} / \mathcal{G}^{3}\right)=\chi\left(\mathcal{M}^{3}\right)
$$

and to represent this by the functional integral

$$
\chi\left(\mathcal{M}^{3}\right)=\int_{\mathcal{A}^{3} / \mathcal{G}^{3}} e_{v, \nabla}\left(\mathcal{A}^{3} / \mathcal{G}^{3}\right) .
$$

Of course, the 'action', i.e. the exponent of (4.29), will contain non-local terms like the curvature tensor $\mathcal{R}_{\mathcal{A} / \mathcal{G}}(4.7)$, as in (4.24). As this is undesirable for a fundamental action, we will introduce auxiliary fields (like those we eliminated in going from (4.17) to (4.24)) to rewrite the action in local form.

Alternatively, we can construct supersymmetric quantum mechanics on $\mathcal{A}^{3} / \mathcal{G}^{3}$ using $\dot{A}+v$ as the section of $T\left(L \mathcal{A}^{3} / \mathcal{G}^{3}\right)$, i.e. we use the action $S_{M, W}$ (3.15) of section 3 and substitute $M \rightarrow \mathcal{A}^{3} / \mathcal{G}^{3}$ and $W \rightarrow C S(A)$. This will give us a (non-covariant) $(3+1)$-dimensional gauge theory on $M \times S^{1}$ (in fact, the $(3+1)$ decomposition of Donaldson theory, see [41, 1] and [18] for details). However, 
from the general arguments of section 3 we know that only the constant Fourier modes will contribute, so that one is left with an effective three-dimensional action which is identical to the one obtained by the first method.

Irrespective of how one chooses to go about constructing the action (there are still further possibilities, see e.g. [13, 14, [17]), it reads

$$
\begin{aligned}
S_{\mathcal{M}} & =\int_{M}\left(B_{1} F_{A}+\alpha B_{1} * B_{1} / 2+d_{A} u * d_{A} u / 2-d_{A} \bar{\phi} * d_{A} \phi+\bar{\psi} d_{A} \psi\right) \\
& +\left(u[\psi, * \bar{\psi}]+\eta d_{A} * \psi+\bar{\eta} d_{A} * \bar{\psi}+\bar{\phi}[\psi, * \psi]-\alpha \phi[\bar{\psi}, * \bar{\psi}] / 2\right) .
\end{aligned}
$$

$u$ is a scalar field, and as in supersymmetric quantum mechanics we have denoted the field $\chi$ of the Mathai-Quillen formula by $\bar{\psi}$. The rest should look familiar. Superficially, this action is very similar to that of Donaldson theory. There is a Gaussian constraint onto flat connections, the tangents $\psi$ have to satisfy the linearized flatness equations, and there are cubic interaction terms involving the scalar fields $\phi, \bar{\phi}$ and $u$. However, there is one important difference, namely that there is a perfect symmetry between $\psi$ and $\bar{\psi}$. As in supersymmetric quantum mechanics, both represent tangent vectors, we also see that both are gauge fixed to be horizontal, and both have to be tangent to $\mathcal{M}^{3}$. In particular, therefore, there will be an equal number of $\psi$ and $\bar{\psi}$ zero modes and we have the possibility of obtaining a non-zero result even if $\operatorname{dim}\left(\mathcal{M}^{3}\right) \neq 0$. This is reassuring as we, after all, expect to find $Z\left(S_{\mathcal{M}}\right)=\chi\left(\mathcal{M}^{3}\right)$. Let us now show that this is indeed the case.

- First of all integration over $\eta$ and $\bar{\eta}$ forces $\psi$ and $\bar{\psi}$ to be horizontal, $h_{A} \psi=$ $\psi, h_{A} \bar{\psi}=\bar{\psi}$, i.e. to represent tangent vectors to $\mathcal{A}^{3} / \mathcal{G}^{3}$

- Setting $\alpha=1$, integration over $\bar{\phi}$ yields $\phi=-G_{A}^{0} *[\psi, * \psi]$, giving rise to a term

$$
\left\langle *[\bar{\psi}, * \bar{\psi}], G_{A}^{0} *[\psi, * \psi]\right\rangle / 2
$$

in the action

- The equation of motion for $u$ reads

$$
u=G_{A}^{0} *[\psi, * \bar{\psi}]
$$

and plugging this back into the action one obtains a term

$$
\left\langle *[\psi, * \bar{\psi}], G_{A}^{0} *[\psi, * \bar{\psi}]\right\rangle / 2
$$

- This combination of Greens function is precisely that appearing in the formula (4.7) for the Riemann curvature tensor $\mathcal{R}_{\mathcal{A} / \mathcal{G}}$. Thus we have already reduced the action to the form $S_{\mathcal{M}}=\mathcal{R}_{\mathcal{A} / \mathcal{G}}+$ 'something' and we expect the 'something' to be the contribution (4.10) to $\mathcal{R}_{\mathcal{M}}$ (4.8) quadratic in the extrinsic curvature $K_{\mathcal{M}}$. 
- To evaluate the integral over the remaining fields $A, \psi$, and $\bar{\psi}$ we expand them about their classical configurations which we can take to be flat connections $A_{c}$ and their tangents (because of $\alpha$-independence). By standard arguments we may restrict ourselves to a one-loop approximation and to this order the remaining terms in the action become

$$
\int_{M}\left(d_{A_{c}} A_{q} * d_{A_{c}} A_{q} / 2+\left[\bar{\psi}_{c}, \psi_{c}\right] A_{q}\right)
$$

- Finally, integration over $A_{q}$ yields

$$
\left\langle\left[\bar{\psi}_{c}, \psi_{c}\right], G_{A_{c}}^{2}\left[\bar{\psi}_{c}, \psi_{c}\right]\right\rangle / 2
$$

which we recognize to be precisely the contribution (4.10) of $K_{\mathcal{M}}$. Thus we have reduced the action (4.30) to $\mathcal{R}_{\mathcal{M}}$, expressed in terms of the classical configurations $A_{c}, \psi_{c}$ and $\bar{\psi}_{c}$. We are now on familiar ground (see e.g. (2.25,3.20) and know that evaluation of this finite dimensional integral gives

$$
Z\left(S_{\mathcal{M}}\right)=\chi(\mathcal{M})
$$

This calculation also illustrates how the Gauss-Codazzi equations emerge from the Mathai-Quillen form in general. Guided by this example it is now straightforward to perform the analogous manipulations in the finite dimensional case (section 2) and in supersymmetric quantum mechanics (section 3).

We end this $3 d$ example with the remark that, by a result of Taubes [26], the partition function of (2.24) formally equals the Casson invariant of $M$ if $M$ is a homology three-sphere [13. This, combined with the above considerations, has led us to propose $\chi(\mathcal{M})$ as a candidate for the definition of the Casson invariant of more general three-manifolds (see [17] for some preliminary considerations).

The simplest example to consider in two dimensions is the analogue of Donaldson theory, i.e. a topological field theory describing intersection theory on a moduli space $\mathcal{M}^{2}$ of flat connections in two dimensions. Instead of the bundle $\mathcal{E}_{+}$ with standard fibre $\Omega_{+}^{2}(M, \mathbf{g})$ (4.16) we choose the bundle $\mathcal{E}_{0}$ (4.15) with standard fibre $\Omega^{0}(M, \mathbf{g})$. This will have the effect of replacing the self-dual two-forms $B_{+}$ and $\chi_{+}$of Donaldson theory by zero-forms $B_{0}$ and $\chi_{0}$. A natural section of $\mathcal{E}_{0}$ is $s(A)=* F_{A}$ with zero locus $\mathcal{M}^{2}$. This results in the trading of $\left(F_{A}\right)_{+}$and its linearization $\left(d_{A} \psi\right)_{+}$for $F_{A}$ and its linearization $d_{A} \psi$ in the action (4.17). With this dictionary in mind the action is precisely the same as that of Donaldson theory. It is also the $2 d$ version of (4.22) and we have thus just completed the construction of

Example $5 X=\mathcal{A}^{2} / \mathcal{G}^{2}, E=\mathcal{E}_{0}, s=* F_{A}$

The fundamental reason for why this theory is so similar to Donaldson theory is that in both cases the deformation complex is short so that one will find essentially the same field content. In three dimensions, on the other hand, the deformation 
complex is longer by one term and this is reflected in the appearance of the scalar field $u$ in (4.30).

Again, the partition function, i.e. the regularized Euler number of $\mathcal{E}_{0}$, will vanish when $\operatorname{dim}\left(\mathcal{M}^{2}\right) \neq 0$. But, none too surprisingly, there also exist analogues of the Donaldson polynomials, the observables of Donaldson theory, which come to the rescue in this case. Life in two dimensions is easier than in four, and the corresponding intersection numbers have indeed been calculated recently by Thaddeus [42] using powerful tools of conformal field theory and algebraic geometry (see also [13, 44]).

As our final example let us consider a topological gauge theory representing the Euler characteristic of $\mathcal{M}^{2}$. As mentioned above, $* F_{A}$ is not a vector field on $\mathcal{A}^{2} / \mathcal{G}^{2}$, so that it is not immediately obvious which section of $T \mathcal{A}^{2} / \mathcal{G}^{2}$ to choose. The dimensional reduction of the action (4.30) suggests, that the right base space to consider is $X=\mathcal{A}^{2} \times \Omega^{0}(M, \mathbf{g})$, where the second factor represents the third component $\rho$ of $A$. Then a possible section of $T X$ is $V(A, \rho)=\left(* d_{A} \rho, * F_{A}\right)$ whose zero locus (for irreducible $A$ ) is indeed precisely the space of flat connections. But this is not the complete story yet. The problem is, that $* d_{A} \rho$ is only horizontal if $A$ is flat $\left(d_{A}^{*} * d_{A} \rho=\left[* F_{A}, \rho\right]\right)$. Thus, one possibility is to use a delta function instead of a Gaussian constraint onto flat connections $(\alpha=0)$. This action can be found in [17]. Alternatively, one might attempt to replace $* d_{A} \rho$ by $h_{A} * d_{A} \rho$. This necessitates the introduction of additional auxiliary fields to eliminate the non-locality of $h_{A}$, and a more detailed investigation of this possibility is left to the reader.

\section{Acknowledgements}

I wish to thank R. Gielerak and the whole organizing committee for inviting me to lecture at this School. Thanks are also due to all the participants for creating such a stimulating atmosphere, and to Jaap Kalkman for sending me [23]. Finally, I wish to acknowledge the financial support of the Stichting FOM.

\section{References}

[1] E. Witten, Topological quantum field theory, Commun. Math. Phys. 117 (1988) 353.

[2] E. Witten, Topological sigma models, Commun. Math. Phys. 118 (1988) 411.

[3] E. Witten, Quantum field theory and the Jones polynomial, Commun. Math. Phys. 121 (1989) 351.

[4] E. Witten, On the structure of the topological phase of two dimensional gravity, Nucl. Phys. B340 (1990) 281.

[5] J. Distler, 2D quantum gravity, topological field theory and the multicritical matrix models, Nucl. Phys. B342 (1990) 523. 
[6] R. Dijkgraaf, E. Verlinde and H. Verlinde, Notes on topological string theory and $2 d$ quantum gravity, in String Theory and Quantum gravity, Proceedings of the Trieste Spring School 1990, M. Green et al. eds. (World Scientific, 1991).

[7] R. Dijkgraaf, Intersection theory, integrable hierarchies and topological field theory, Lectures at the 1991 Cargèse Summer School on New Symmetry Principles in Quantum Field Theory, preprint IASSNS-HEP-91/91.

[8] E. Witten, Two dimensional gravity and intersection theory on moduli space, Surveys in Diff. Geom. 1 (1991) 243.

[9] D. Birmingham, M. Blau, M. Rakowski and G. Thompson, Topological Field Theory, Physics Reports 209 Nos. $4 \& 5$ (1991) 129-340.

[10] V. Mathai and D. Quillen, Superconnections, Thom classes and equivariant differential forms, Topology 25 (1986) 85.

[11] M.F. Atiyah and L. Jeffrey, Topological Lagrangians and cohomology, J. Geom. Phys. 7 (1990) 120.

[12] S.K. Donaldson, A polynomial invariant for smooth four-manifolds, Topology 29 (1990) 257.

[13] E. Witten, Topology changing amplitudes in $2+1$ dimensional gravity, Nucl. Phys. B323 (1989) 113.

[14] D. Birmingham, M. Blau and G. Thompson, Geometry and quantization of topological gauge theories, Int. J. Mod. Phys. A5 (1990) 4721.

[15] D. Montano and J. Sonnenschein, The topology of moduli space and quantum field theory, Nucl. Phys. B313 (1989) 258; J. Sonnenschein, Topological quantum field theories, moduli spaces and flat gauge connections, Phys. Rev. D42 (1990) 2080.

[16] G. Thompson, Introduction to topological field theory, lectures at the Summer School on High-Energy Physics and Cosmology, Trieste, July 1991.

[17] M. Blau and G. Thompson, $N=2$ topological gauge theory, the Euler characteristic of moduli spaces, and the Casson invariant, preprint NIKHEF-H/91-28, MZ-TH/91-40 (November 1991), submitted to Commun. Math. Phys.

[18] M. Blau and G. Thompson, Topological gauge theories from supersymmetric quantum mechanics on spaces of connections, Mainz preprint MZ-TH/91-42 (December 1991), submitted to Phys. Lett. B.

[19] E. Witten, The $N$ matrix model and gauged WZW models, IAS preprint (June 1991).

[20] P.S. Aspinwall and D.R. Morrison, Topological field theory and rational curves, Oxford University preprint OUTP-91-32P.

[21] R. Bott and L.W. Tu, Differential Forms in Algebraic Topology, Springer, New York, 1986.

[22] D. Birmingham, The algebraic structure of cohomological field theory, lectures at this School. 
[23] J. Kalkman, BRST model for equivariant cohomology and representatives for the equivariant Thom class, Department of Mathematics, University of Utrecht, preprint Nr. 708 (January 1992).

[24] J.-M. Bismut, The Atiyah-Singer theorems. A probabilistic approach. I. The index theorem, J. Funct. Anal. 57 (1984) 56; II. The Lefschetz fixed point formulas, ibid. 57 (1984) 329.

[25] J.-M. Bismut, Index theorem and equivariant cohomology on the loop space, Commun. Math. Phys. 98 (1985) 213; Localization formulas, superconnections, and the index theorem for families, ibid. 103 (1986) 127.

[26] C.H. Taubes, Casson's invariant and Riemannian geometry, J. Diff. Geom. 31 (1990) 547.

[27] E. Witten, Supersymmetry and Morse theory, J. Diff. Geom. 17 (1982) 661.

[28] E. Witten, Constraints on supersymmetry breaking, Nucl. Phys. B202 (1982) 253.

[29] L. Alvarez-Gaumé, Supersymmetry and the Atiyah-Singer index theorem, Commun. Math. Phys. 90 (1983) 161.

[30] L. Alvarez-Gaumé, Supersymmetry and index theory, in Supersymmetry, NATO Adv. Study Institute, eds. K. Dietz et al. (Plenum, New York, 1985).

[31] D. Friedan and P. Windey, Supersymmetric derivation of the Atiyah-Singer index theorem, Nucl. Phys. B235 (1984) 395.

[32] N.S. Narasimhan and T.R. Ramadas, Geometry of $S U(2)$ gauge fields, Commun. Math. Phys. 67 (1979) 121.

[33] I.M. Singer, Some remarks on the Gribov ambiguity, Commun. Math. Phys. 60 (1978) 7 .

[34] O. Babelon and C.M. Viallet, The Riemannian geometry of the configuration space of gauge theories, Commun. Math. Phys. 81 (1981) 515.

[35] D. Groisser and T.H. Parker, The Riemannian geometry of the Yang-Mills moduli space, Commun. Math. Phys. 112 (1987) 663.

[36] D.S. Freed and K.K. Uhlenbeck, Instantons and Four-Manifolds, M.S.R.I. publications, Springer, New York, 1984; S.K. Donaldson and P.B. Kronheimer, The Geometry of Four-Manifolds, Oxford Mathematical Monographs, Clarendon Press, Oxford, 1990.

[37] M.F. Atiyah, Topological quantum field theories, Publ. Math. IHES 68 (1988) 175.

[38] S. Axelrod, Geometric quantization of Chern-Simons gauge theory, Ph.D. thesis, Princeton Univ. (1991).

[39] M.F. Atiyah and I.M. Singer, Dirac operators coupled to vector potentials, Proc. Nat'l Acad. Sci. (USA) 81 (1984) 2597.

[40] L. Baulieu and I.M. Singer, Topological Yang-Mills symmetry, Nucl. Phys. Proc. Suppl. 5B (1988) 12. 
[41] M.F. Atiyah, New invariants of three and four dimensional manifolds, in: The mathematical heritage of Hermann Weyl, Proc. Symp. Pure Math. 48, R. Wells et al. (eds.), Am. Math. Soc., Providence, 1988.

[42] M. Thaddeus, Conformal field theory and the moduli space of stable bundles, Oxford University preprint.

[43] E. Witten, Quantum gauge theories in two dimensions, Commun. Math. Phys. 141 (1991) 153.

[44] S.K. Donaldson, Glueing problems in Yang-Mills theory, 30. Arbeitstagung Bonn, 14.-20. June 1991. 